\title{
Evaluación de Modelos de Turbulencia para el Flujo de Aire en un Difusor Transónico
}

\author{
Tolentino-Masgo, San Luis B. ${ }^{1,2, * \text { iD }}$ \\ ${ }^{1}$ Universidad Nacional Experimental Politécnica “Antonio José de Sucre” Vice-Rectorado Puerto Ordaz, Centro de Estudios Energéticos, \\ Bolivar, Venezuela \\ ${ }^{2}$ Universidad Nacional de Ingeniería, Grupo de Modelamiento Matemático y Simulación Numérica, Lima, Perú
}

\begin{abstract}
Resumen: El campo de flujo con presencia de ondas de choque, turbulencia y separación de flujo se estudia mediante el empleo de equipos experimentales y puede reproducirse utilizando códigos computacionales. En el presente trabajo el objetivo es evaluar cinco modelos de turbulencia: SST $\boldsymbol{k}-\boldsymbol{\omega}, \boldsymbol{k}-\boldsymbol{e}$ estándar, $\boldsymbol{k}-\boldsymbol{\omega}$ estándar, $\boldsymbol{k}-\boldsymbol{k} \boldsymbol{l}-\boldsymbol{\omega}$ de transición y RSM, para el flujo de aire con presencia de ondas de choque en un difusor transónico. Se realizaron simulaciones numéricas 2D del campo de flujo aplicando el modelo RANS en el código ANSYSFluent, el cual aplica el método de volumen finito. Se emplearon las ecuaciones gobernantes: conservación de la masa, cantidad de movimiento, energía y de estado; para la viscosidad en función de la temperatura la ecuación de Sutherland. Además, se consideró el análisis basado en densidad para un fluido compresible. Se obtuvieron resultados numéricos de perfiles de presión estática en las paredes y de la velocidad en diferentes posiciones en el difusor, así como, del campo de densidad, número de Mach, presión estática, velocidad y temperatura estática, para las relaciones de presión $\boldsymbol{r p}=\mathbf{0 , 8 2}$ y $\boldsymbol{r p}=\mathbf{0 , 7 2}$, los cuales fueron comparados con datos experimentales publicados en la literatura. Se concluye que el modelo de turbulencia SST $\boldsymbol{k}-\boldsymbol{\omega}$ de Menter se ajusta más a los datos experimentales de presión y de velocidad para el flujo con presencia de ondas de choque.
\end{abstract}

Palabras clave: Difusor transónico, Flujo de aire, Modelo de turbulencia, Onda de choque, Separación de flujo

\section{Evaluation of Turbulence Models for the Air Flow in a Transonic Diffuser}

\begin{abstract}
The flow field with the presence of shock waves, turbulence and flow separation is studied through the use of experimental equipment and can be reproduced using computational codes. In the present work the objective is to evaluate five turbulence models: SST $\boldsymbol{k}-\boldsymbol{\omega}, \boldsymbol{k}-\boldsymbol{e}$ standar, $\boldsymbol{k}-\boldsymbol{\omega}$ standar, transition $\boldsymbol{k}-\boldsymbol{k} \boldsymbol{l}-\boldsymbol{\omega}$ and RSM, for the air flow with the presence of shock waves in a diffuser transonic. 2D numerical simulations of the flow field were performed by applying the RANS model in the ANSYS-Fluent code, which applies the finite volume method. The governing equations were used: conservation of mass, momentum, energy, and state; for viscosity versus temperature the Sutherland equation. In addition, density-based analysis for a compressible fluid was considered. Numerical results of static pressure profiles in the walls and of the velocity in different positions in the diffuser were obtained, as well as, of the density field, Mach number, static pressure, velocity and static temperature, for the pressure ratios $\boldsymbol{r} \boldsymbol{p}=\mathbf{0 , 8 2}$ and $\boldsymbol{r} \boldsymbol{p}=\mathbf{0 , 7 2}$, which were compared with experimental data published in the literature. It is concluded that the Menter SST $\boldsymbol{k}-\boldsymbol{\omega}$ turbulence model is more in tune with the experimental pressure and velocity data for the flow with the presence of shock waves.
\end{abstract}

Keywords: Transonic diffuser, Air flow, Turbulence model, Shock wave, Flow separation

\section{INTRODUCCIÓN}

Las ondas de choque y la interacción de la capa límite turbulenta están presente en los flujos de gas a alta velocidad, para un flujo interno o externo. Una onda de choque se produce por un cambio brusco de la presión de un fluido gas

sanluist@gmail.com

Recibido: $23 / 02 / 2019$

Aceptado: $11 / 03 / 2020$

Publicado: 31/04/2020

10.33333/rp.vol45n 1.03 en contacto con un cuerpo que se desplaza a una velocidad mayor a la velocidad del sonido. En la región del choque, las magnitudes termodinámicas de temperatura, densidad, entre otros parámetros, varían drásticamente.

En la literatura están reportados trabajos sobre la capa límite de flujo compresible (White, 1991), donde las curvas teóricas de la ley de pared y de la ley logaritmo son comparados con datos experimentales de velocidad (Lee et al., 1969); la capa límite con diferentes condiciones de gradiente de presión (Schlichting, 1979); la teoría de las ondas de choque normal, oblicuas y de las ondas expansivas de Prandtl-Meyer (Anderson, 2007; White, 2008); la teoría de la turbulencia 
(Kármán, 1937); el modelado de la turbulencia (Wilcox, 2006); así como, el principio y aplicaciones de la dinámica de fluidos computacional (CFD, por sus siglas en inglés) (Blazek, 2005; Anderson et al., 2012).

Una de las maneras de estudiar el comportamiento del campo de flujo compresible a velocidad subsónica, transónica y supersónica es mediante el empleo de equipos experimentales, tales como los difusores y las toberas; donde, por las variaciones de la densidad del flujo, las formas de las ondas de choque y las turbulencias son captadas en imágenes mediante la técnica Schlieren. La técnica Schlieren fue propuesto por el físico alemán August Toepler en 1864 (Krehl y Engemann, 1995) y fue el primero en visualizar la geometría de la onda de choque; y esta técnica se emplea de manera recurrente para visualizar el campo de flujo.

Un tipo de difusor transónico, clasificado como modelo G, también conocido como difusor Sajben, inicialmente fue empleado por Sajben et al. (1981) para realizar ensayos experimentales de flujos transónicos con presencia de choque débil para el flujo con $r_{p}=0,82$ para Mach 1,235, y choque fuerte para el flujo con $r_{p}=0,72$ para Mach 1,353. Las configuraciones de las formas de las ondas de choque fueron captadas con la técnica Schlieren, así como, el inicio de la separación de flujo y la recirculación de flujo aguas abajo después del choque. Donde, para el choque fuerte se presentó un frente de onda normal y choque oblicuo adyacente a la pared superior.

Posteriormente, el difusor transónico fue empleado por Salmon et al. (1983), quien utilizó un velocímetro laser Doppler para medir la velocidad del flujo en diferentes regiones. Bogar et al. (1983) estudió el flujo temporal e inestable en función del número de Mach en secciones transversales a lo largo del difusor; además, Bogar (1986) realizó también mediciones con un velocímetro laser Doppler al campo de flujo.

Se señalan a algunos autores de trabajos relevantes que han empleado la geometría del difusor transónico Sajben para simular el campo de flujo aplicando la CFD, para evaluar diferentes modelos de turbulencia y comparar los resultados numéricos con datos experimentales: Liou et al. (1981) emplearon el método hibrido MacCormack's y el modelo original de Wilcox-Rubesin para representar la viscosidad de remolino; Hsieh et al. (1987) utilizaron una versión modificada del método hibrido MacCormack`s, así como, el modelo de Wilcox-Rubesin, y simularon el campo de flujo con el código NASA-ARC-Cray/XMP; Georgiadis et al. (1994) emplearon el código PARC, y para la simulación de la turbulencia del flujo utilizaron los modelos: Thomas, Baldwin-Lomax, MMLT, Chien $k-\varepsilon$ y Speziale $k-\varepsilon$; Bush et al. (1998) emplearon tres códigos computacionales: el código NPARC y el modelo de turbulencia BaldwinLomax, y para los códigos WIND y NXAIR recurrieron al modelo Spalart-Allmaras; Yoder y Georgiadis (1999) emplearon el código WIND y el modelo de turbulencia de Chien $k-\epsilon$; Xiao y Tasai (2003) utilizaron el modelo $k-$ $\omega$; Wüthrich (2007) simuló el flujo con el código OpenFOAM; Vlahostergios y Yakinthos (2015) utilizaron los modelos RSM, NLEVM y LEVM; Biswas y Jimbo, (2015) emplearon un modelo de turbulencia de dos ecuaciones, $k-$ $\varepsilon$; Liu et al. (2016) utilizaron los modelos de turbulencia: Spalart-Allmaras, $k-\varepsilon$ estándar, RNG $k-\varepsilon, k-\varepsilon$ realizable, $k-\omega$ estándar, SST $k-\omega, \overline{v^{2}}-f$ y RSM, y el código ANSYS-Fluent. Además, Tolentino y Caraballo (2017) emplearon el modelo SST $k-\omega$, y para la simulación el código ANSYS-Fluent.

La CFD ha alcanzado gran aceptación en las simulaciones del campo de flujo compresible, empleando el modelo de las ecuaciones de Navier-Stokes de número de Reynolds promedio (RANS, por sus siglas en inglés), el cual requiere de modelos de turbulencia.

Los modelos de turbulencia permiten reproducir la turbulencia del flujo en dominios computacionales que tienen geometrías complejas, por lo cual, determinan las magnitudes de los parámetros termodinámicos que no es posible obtener por medios experimentales. Son tomados en cuenta de acuerdo al comportamiento del régimen de flujo, y de las condiciones de gradientes adversos de presión y de otros parámetros termodinámicos del fluido, dentro y fuera de la capa límite, antes y después del choque. Con el pasar de los años, se han formulado nuevos modelos de turbulencia y, en base a esto, de manera recurrente, se continúa evaluando diferentes modelos de turbulencia para determinar cuáles de los modelos tienen las mejores prestaciones en reproducir diferentes condiciones del régimen del flujo para diferentes casos en específicos.

En el presente trabajo, el objetivo es evaluar cinco modelos de turbulencia para el flujo de aire para $r_{p}=0,82$ y $r_{p}=$ 0,72, para la geometría de un difusor transónico experimental, modelo G (Sajben et al., 1981), con el fin de determinar cuál de los modelos de turbulencia empleados se ajusta más a los datos experimentales de presión y de velocidad que están reportados en la literatura. Así como, determinar los espesores de la onda de choque débil y onda de choque fuerte que se presentan en el flujo. Para lograr la meta trazada, se realizó las simulaciones en 2D del campo de flujo y se comparó los resultados numéricos con los datos experimentales de presión estática y de velocidad. En base a los resultados, el modelo de turbulencia SST $k-\omega$ (Menter, 1994) se ajustó más a los datos experimentales.

En la sección 2 se presentan las ecuaciones empleadas, el dominio computacional, el dominio mallado y el método de solución computacional. En la Sección 3 se exponen los resultados obtenidos y las discusiones. En la sección 4 se exponen las conclusiones del análisis realizado.

\section{METODOLOGÍA}

\subsection{Fundamento matemático}

En el presente trabajo, para la solución numérica del campo de flujo compresible se empleó el modelo RANS.

Las ecuaciones gobernantes empleadas, son: la Ecuación (1) de la conservación de la masa, la Ecuación (2) de la cantidad de movimiento, la Ecuación (3) de la conservación de la 
energía y la Ecuación (4) de estado. La simulación computacional se consideró para flujo en estado estacionario, y las ecuaciones antes mencionadas en su forma compacta, se expresan como

$$
\nabla \cdot\left(\rho u_{i}\right)=0
$$

Donde, la densidad es $\rho$; y la velocidad $u$.

$$
\nabla \cdot\left(\rho u_{i} u_{j}\right)=-\nabla p+\nabla \cdot(\overline{\bar{\tau}})+\nabla \cdot\left(-\rho \overline{u_{\imath}^{\prime} u_{j}^{\prime}}\right)
$$

Donde, la presión es $p$; el tensor de tensiones $\overline{\bar{\tau}}$. Esta Ecuación (2) está cerrada, ya que incluye el término de tensiones de Reynolds, que se expresa como $-\rho \overline{u_{l}^{\prime} u_{\jmath}^{\prime}}$.

$$
\nabla \cdot\left(u_{i}(\rho E+p)\right)=\nabla \cdot\left(k_{e f f} \nabla T+\left(\overline{\bar{\tau}}_{e f f} \cdot u_{i}\right)\right)
$$

Donde, la energía total es $E$, la temperatura $T$, la conductividad térmica efectiva $k_{e f f}$, y el tensor de tensiones efectivo $\overline{\bar{\tau}}_{\text {eff }}$.

$$
p=\rho R T
$$

Donde, la constante del gas es $R$. Por despeje, la densidad se expresa como $\rho=p / R T$.

La Ecuación (5) de relaciones de presión, y la Ecuación (6) de relaciones de temperatura, se expresan como

$$
\begin{aligned}
& \frac{p_{0}}{p}=\left(1+\frac{\gamma-1}{2} M^{2}\right)^{\frac{\gamma}{\gamma-1}} \\
& \frac{T_{0}}{T}=1+\frac{(\gamma-1)}{2} M^{2}
\end{aligned}
$$

Donde, la presión total es $p_{0}$, la temperatura total $T_{0}$, la relación de calor específico $\gamma$; y el número de Mach $M$, el cual es el parámetro dominante para flujo compresible. El rango de número de Mach, se clasifica como: para flujo incompresible $M<0,3$; flujo subsónico $0,3<M<0,8$; flujo transónico $0,8<M<1,2$; flujo supersónico $1,2<$ $M<3$; flujo hipersónico $M>3$. Para el flujo con velocidad sónica $M=1$ (White, 2008). Es importante acotar, White (2008) considera que a partir de un valor mayor de Mach 3 el flujo es hipersónico y Anderson (2007) considera a partir de Mach 5.

La Ecuación (7), es la variación de la viscosidad para gases en función de la temperatura, se expresa como un aproximado de acuerdo a la ley de Sutherland (White, 2008)

$$
\frac{\mu}{\mu_{0}}=\left(\frac{T}{T_{0}}\right)^{\frac{3}{2}} \frac{T_{0}+S}{T+S}
$$

Siendo la viscosidad de referencia $\mu_{0}=1,716 \mathrm{~kg} /(\mathrm{m} . \mathrm{s})$, la temperatura de referencia $T_{0}=273,11 \mathrm{~K}$, y la temperatura efectiva $S=110,56 \mathrm{~K}$.

Los cinco modelos de turbulencia empleados en la simulación del flujo, los cuales fueron ensamblados de manera individual al modelo RANS, son las siguientes: SST $k-\omega$ (Menter, 1994), $\quad k-e$ estándar (Launder y Spalding, 1972), $k-\omega$ estándar (Wilcox, 1988), $k-k l-\omega$ de transición (Walters y Cokljat, 2008), donde, estos cuatro modelos de turbulencia son modelos de viscosidad turbulenta y están sustentados por la hipótesis de Boussinesq. Y el quinto, el modelo de turbulencia RSM (Launder et al., 1975) para la tensión lineal de presión y efectos de reflexión de la pared (Gibson y Launder, 1978; Launder, 1989), la cual está sustentando en los modelos de tensiones de Reynolds.

Los modelos de turbulencias, son ecuaciones de transporte semiempírica que modelan el mezclado y difusión que se incrementan a causa de remolinos turbulentos, los cuales están en función de la viscosidad del fluido y de la viscosidad turbulenta, entre otras variables. Wilcox (2006) reporta diferentes modelos de turbulencia, y cuyas formulaciones semiempírica están sustentadas en base a las primeras investigaciones de la turbulencia desarrolladas por Kolmogorov (1941) en base a los resultados de Reynolds en 1883.

\subsection{Dominio computacional}

El dominio computacional 2D de la geometría del difusor transónico experimental, modelo G (Sajben et al., 1981), que se consideró para el estudio del flujo, se muestra en la Figura 1. La pared inferior tiene una base recta en toda su longitud, y en la pared superior es recta en los extremos y, en la parte media es curva.

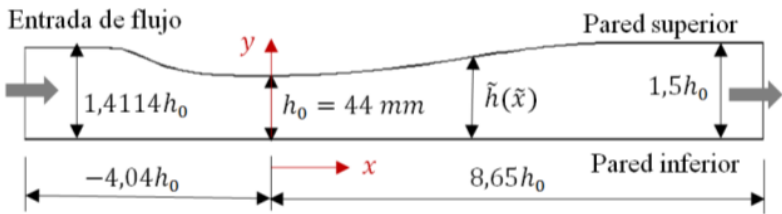

Figura 1. Geometría del dominio computacional del difusor transónico. Construido a partir del esquema reportado por Sajben et al. (1981).

Las restricciones para las condiciones de borde (C.B.) en el dominio computacional se señalan en la Figura 2. Se estableció para dos casos: un caso para el flujo con onda de choque débil y el otro caso para el flujo con onda de choque fuerte.

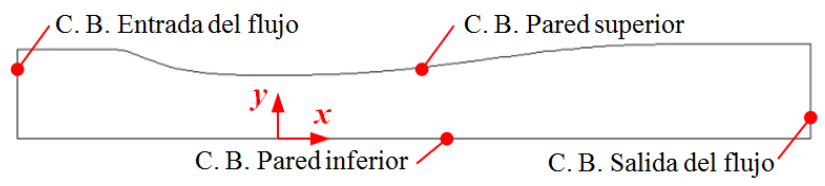

Figura 2. Condiciones de borde en las paredes, en la entrada y salida del flujo en el dominio computacional 2D.

Condiciones de borde aplicadas para el flujo con onda de choque débil, para rp $=0,82$ :

Presión de entrada: $135004,1 \mathrm{~Pa}(19,58$ psi)

Temperatura de entrada: $300 \mathrm{~K}$ (540 R)

Presión de salida: $110664,8 \mathrm{~Pa}(16,05 \mathrm{psi})$

Temperatura de salida: $300 \mathrm{~K}$ (540 R)

Condiciones de borde aplicadas para el flujo con onda de choque fuerte, para $\mathrm{rp}=0,72$ : 
Presión de entrada: 135004,1 Pa (19,58 psi)

Temperatura de entrada: $300 \mathrm{~K}(540 \mathrm{R})$

Presión de salida: 97219,5 Pa (14,1 psi)

Temperatura de salida: $300 \mathrm{~K}$ (540 R)

Para ambos casos, la velocidad del flujo en las paredes es nula, por la condición de no deslizamiento. Además, las paredes se consideraron adiabáticas.

Cabe señalar, los datos de presión para el caso del flujo con onda de choque débil y choque fuerte fueron obtenidos del trabajo de Bogar et al. (1983), y la temperatura total 300 K (540 R) del trabajo de Georgiadis et al. (1994).

\subsection{Mallado del dominio}

El dominio computacional mallado se muestra en la Figura 3. Se aplicó una tecnología de mallado estructurado con celdas cuadriláteras, en la dirección horizontal 179 celdas y en la dirección vertical 100 celdas, para un total de 17900 celdas cuadriláteras; siendo un mallado final luego que se refinó tres veces. Se refinó las regiones adyacentes a las paredes superior e inferior con mayor densidad de celdas, por la presencia del esfuerzo cortante y de la capa límite, así como, se refinó la región donde se presenta la onda de choque. El dominio se discretizó mediante la interacción ICEM-CFD, en la plataforma ANSYS-Meshing.

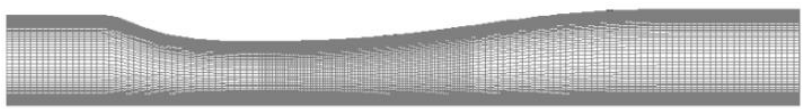

Figura 3. Dominio computacional mallado con 17900 celdas cuadriláteras.

Cabe resaltar, el estudio de independencia de mallado para los tres casos de refinamiento de la malla, consistió en tomar en cuenta como parámetro de control la caída de presión en la pared inferior, para el flujo con $r p=0,82$. Para las soluciones numéricas del refinamiento del mallado, se aplicó el método de solución computacional, considerando los cinco modelos de turbulencia.

En la Tabla 1 se presenta la cantidad de celdas de la malla para los tres casos de estudio: 15300 celdas, 16625 celdas y 17900 celdas; así como las caídas de presión; en la Figura 4 se muestra una gráfica correspondiente a los datos de la Tabla 1.

Se determinó el error porcentual, considerando como valor fijo para la caída de presión para la malla con 17900 celdas, para cada modelo de turbulencia, en función de las presiones con menor densidad de malla. El error porcentual para SST $k-\omega$ arrojó valores menores de $0,00147 \%$, para $k-e$ menor de $0,00034 \%$, para $k-\omega$ menor de $0,00048 \%$, para $k-k l-\omega$ menor de $0,00166 \%$, y para RSM menor de $0,00131 \%$, respectivamente.

En base a los resultados numéricos de los errores porcentuales, las tres mallas están refinadas con alta densidad en las paredes del difusor transónico; donde, la densidad para los tres casos de mallado en un dominio 2D se obtuvo $y^{+}<$ 1. Cabe destacar, es muy común refinar en las regiones de las paredes para el flujo compresible cuando existe separación de flujo y presencia de ondas de choque.

Tabla 1. Densidad de la malla, para el flujo con $r p=0,82$. Las caídas de presión corresponden para la pared inferior, al inicio de la separación del

\begin{tabular}{cccc}
\multicolumn{4}{c}{ flujo. } \\
\hline Celdas: & $\mathbf{1 5 3 0 0}$ & $\mathbf{1 6 6 2 5}$ & $\mathbf{1 7 9 0 0}$ \\
& \multicolumn{3}{c}{ Relación de presión, $\boldsymbol{P} / \boldsymbol{P}_{\mathbf{0}}$} \\
\hline SST $k-\omega$ & 0,4258087 & 0,4258120 & 0,425815 \\
$k-e$ & 0,3911357 & 0,3911364 & 0,391137 \\
$k-\omega$ & 0,4085790 & 0,4085800 & 0,408581 \\
$k-k l-\omega$ & 0,3726328 & 0,3726361 & 0,372639 \\
RSM & 0,3965598 & 0,3965625 & 0,396565 \\
\hline
\end{tabular}

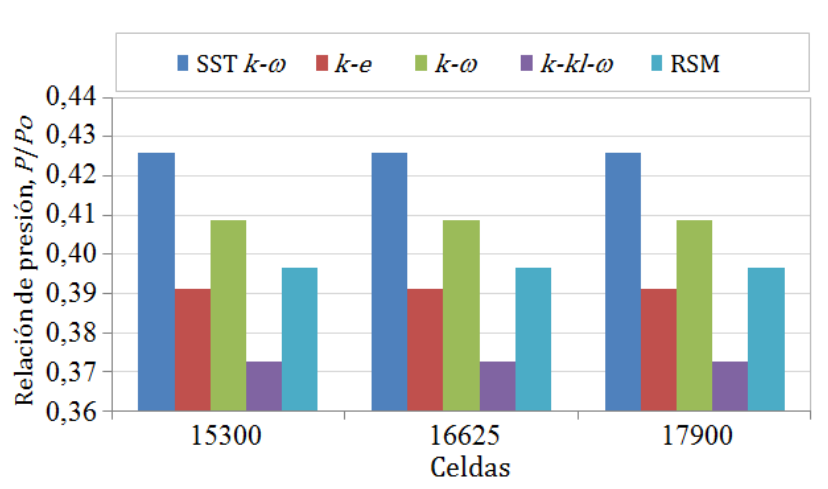

Figura 4. Caídas de presión versus densidad de la malla, evaluados para cinco modelos de turbulencia. Gráfica correspondiente a los datos numéricos de la Tabla 1.

\subsection{Método de solución computacional}

Se empleó el código ANSYS-Fluent Versión 12.1 que aplica el método de volumen finito (MVF) para las simulaciones numéricas. Dentro de las diferentes alternativas de solución, se seleccionó la opción de análisis basado en densidad para fluido compresible; dominio 2D en el plano cartesiano; sustancia de trabajo: aire. Para cada simulación se empleó un solo modelo de turbulencia, en el siguiente orden: SST $k-$ $\omega, k-e$ estándar, $k-\omega$ estándar, $k-k l-\omega$ de transición y RSM. Para la viscosidad del fluido en función de la temperatura se seleccionó la ecuación de Sutherland. En las condiciones del flujo, la turbulencia de la energía cinética y el tipo de disipación específica, se seleccionó la opción: Second Order Upwin. En el monitor residual se determinó para la convergencia numérica, un valor fijo de 0,00001, tanto para continuidad, velocidad y energía. Se obtuvo el rango de 3900-5000 iteraciones para las simulaciones del flujo, para $r p=0,82$ y $r p=0,72$, para los cinco modelos de turbulencias empleados.

Para el procesamiento de datos de la simulación del flujo, se empleó un equipo con las siguientes características: Laptop marca Síragon, modelo M54R, Intel Core 2 Duo, dos procesadores de 1,8 GHz, y memoria RAM de $3 \mathrm{~GB}$.

\section{RESULTADOS Y DISCUSIÓN}

En esta sección, para el régimen del flujo con onda de choque débil para $r p=0,82$ y choque fuerte para $r p=0,72$, se presenta y discuten los resultados numéricos para la densidad, presión estática y velocidad, empleando los cinco modelos de turbulencia: SST $k-\omega, k-e, k-\omega, k-k l-$ $\omega$ y RSM; los cuales son comparados con datos 
experimentales. Así como, los resultados numéricos obtenidos con el modelo de turbulencia SST $k-\omega$ para el número de Mach y la temperatura estática.

\subsection{Campo de densidad y número de Mach}

Las simulaciones numéricas de las distribuciones de la densidad del flujo, para el flujo con relación de presión $r p=$ 0,82 se muestran en la Figura 5, y para $r p=0,72$ en la Figura 6, respectivamente. Donde, los resultados numéricos de las formas de la onda de choque para el campo de densidad son comparadas con las ondas de choque experimentales reportados en el trabajo de Bogar et al. (1983), previo sustento de análisis con la magnitud del número de Mach.

Las variaciones de la densidad en el campo de flujo muestran en que regiones el flujo está comprimido y descomprimido, para el flujo de aire con choque débil, para $r p=0,82$ (Figura 5). Se muestra que la geometría de la forma de la onda de choque es normal en el frente, y tiende a curvarse hacia las adyacencias de las paredes, tal como se aprecia por el gradiente de densidad, y están definidos por las líneas de contorno. Donde se produce la caída de presión, al inicio del choque, la magnitud de la densidad es mínima, al final del choque la magnitud es máxima. Donde se produce la separación del flujo, adyacente a ambas paredes, aguas abajo disminuye la densidad hasta cierta distancia, para luego incrementar su magnitud. Las formas de las ondas de choque débil son similares entre sí para los cinco modelos de turbulencia; para $k-e, k-\omega, k-k l-\omega$ y RSM, la posición de las ondas están desplazados hacia el extremo derecho de la posición para SST $k-\omega$.

Así mismo, para la densidad flujo de aire con choque fuerte para $r p=0,72$ (Figura 6), el perfil de la onda de choque fuerte es más curvada que en el caso anterior, y se observa cómo está configurada, donde en la pared inferior se muestra el desprendimiento de la capa límite, mientras que en la pared superior el desprendimiento de la capa límite es mayor, incluso se tiene la presencia de choque oblicuo y reflejado. En la pared superior, aguas abajo del inicio de la separación del flujo, se muestra como el flujo se separa, por lo cual, está acompañado de una recirculación del flujo por los gradientes adversos de la presión en esa región. Las posiciones de las ondas están ligeramente cercas entre sí para SST $k-\omega, k-$ $\omega$ y RSM, y desplazados hacia el extremo derecho para $k-e$ y $k-k l-\omega$.

Al comparar, ambos casos del flujo, para $r p=0,82$ (Figura 5) y $r p=0,72$ (Figura 6), el perfil geométrico de la pared superior de la sección divergente del difusor contribuye con mayor intensidad en el desprendimiento de la capa límite, con respecto a la pared inferior, además, las ondas de choque no tienen una posición fija, sino, están desfasados unos respecto del otro.

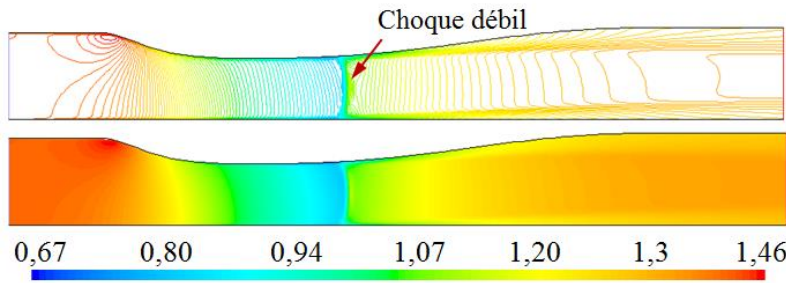

a) $\operatorname{SST} k-\omega$

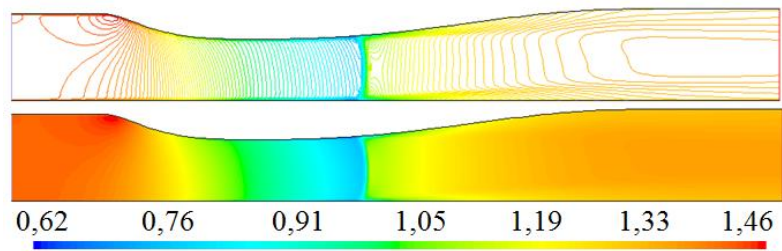

b) $k-e$

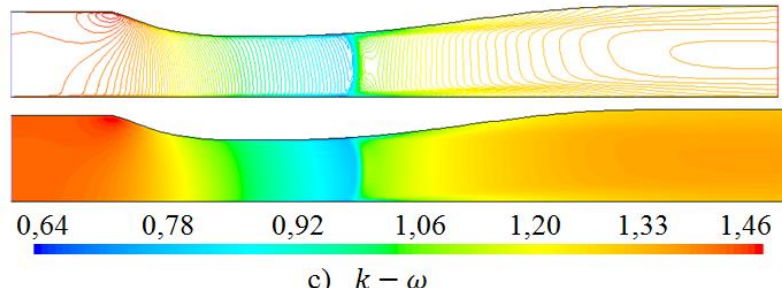

c) $k-\omega$

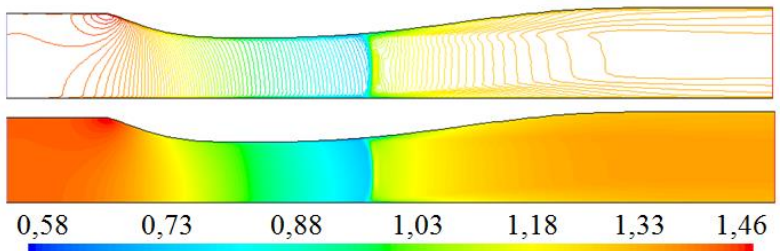

d) $k-k l-\omega$

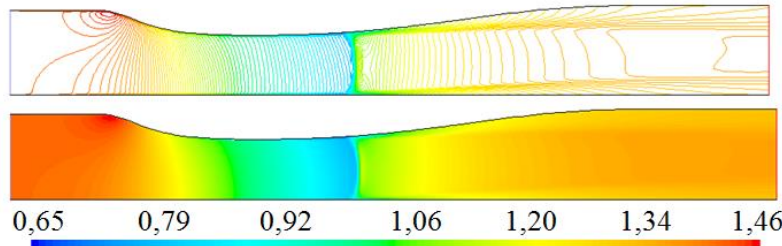

e) RSM

Figura 5. Campo de densidad $\left(\mathrm{kg} / \mathrm{m}^{3}\right)$, para el flujo con $\mathrm{rp}=0,82$. Formas de ondas de choque para cinco modelos de turbulencia.

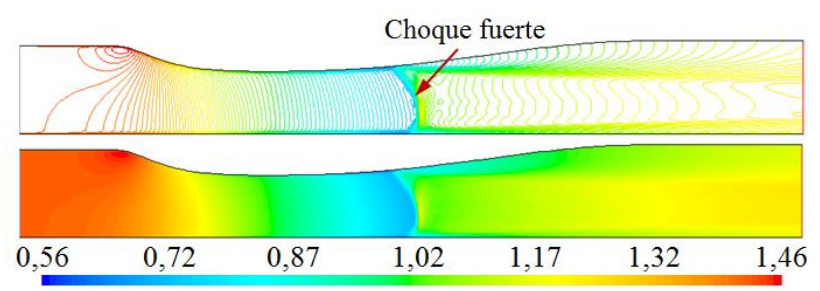

a) $\operatorname{SST} k-\omega$

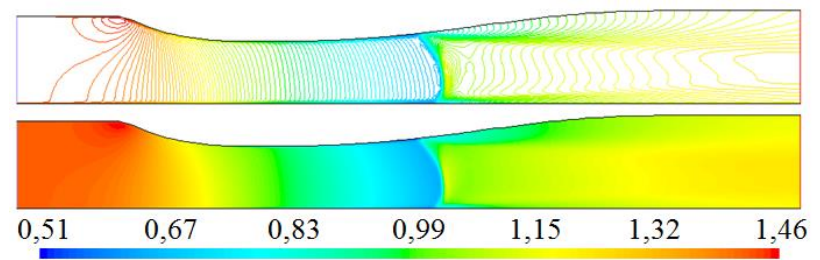

b) $k-e$ 


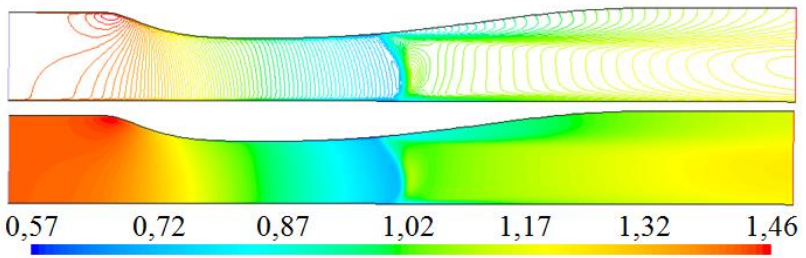

c) $k-\omega$

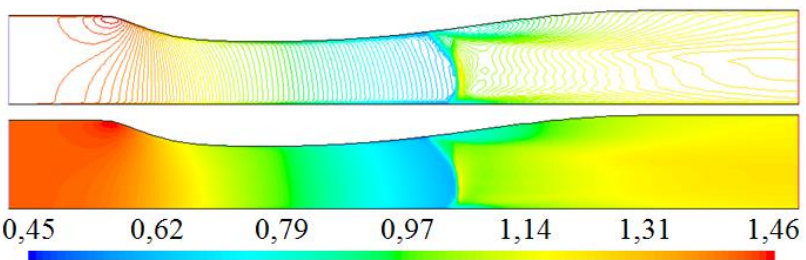

d) $k-k l-\omega$

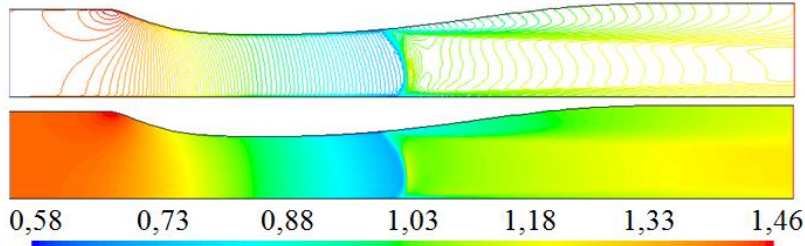

e) RSM

Figura 6. Campo de densidad $\left(\mathrm{kg} / \mathrm{m}^{3}\right)$, para el flujo con $\mathrm{rp}=0,72$. Formas de ondas de choque para cinco modelos de turbulencia.

Los perfiles de densidad evaluados a lo largo de todo el difusor transónico, para los cinco modelos de turbulencia, para la altura de $y=22 \mathrm{~mm}$, lo cual corresponde la altura media de la garganta, se muestran para $r p=0,82$ en la Figura 7 y para $r p=0,72$ enla Figura 8 .

El comportamiento de las trayectorias de las curvas muestra la variación de la magnitud de la densidad antes y después del choque. Para el flujo con $r p=0,82$, el choque para $k-$ $e, k-\omega, k-k l-\omega$ y RSM están desplazados al extremo derecho de SST $k-\omega$. Para el flujo con $r p=0,72$, son casi coincidentes las posiciones del choque para SST $k-\omega, k-$ $\omega$ y RSM, y desplazados hacia el extremo derecho $k-e$ y $k-k l-\omega$.

Para el flujo con $r p=0,82$, la densidad en la salida del difusor está alrededor del valor estimado de $1,35 \mathrm{~kg} / \mathrm{m}^{3}$; y para el flujo con $r p=0,72$, un valor estimado de $1,22 \mathrm{~kg} / \mathrm{m}^{3}$, respectivamente.

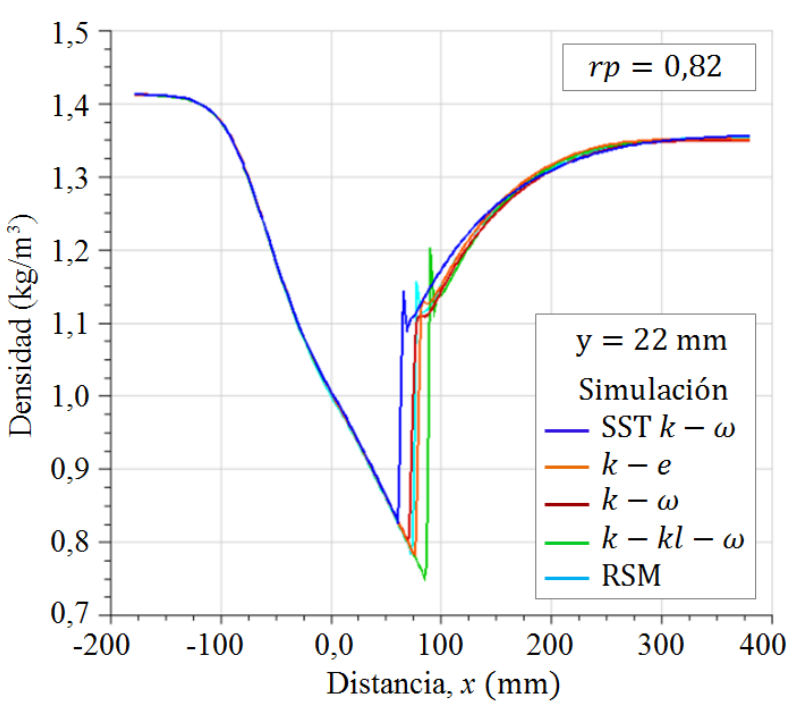

Figura 7. Perfiles de densidad, para el flujo con $\mathrm{rp}=0,82$; obtenido en la altura $\mathrm{y}=22 \mathrm{~mm}$.

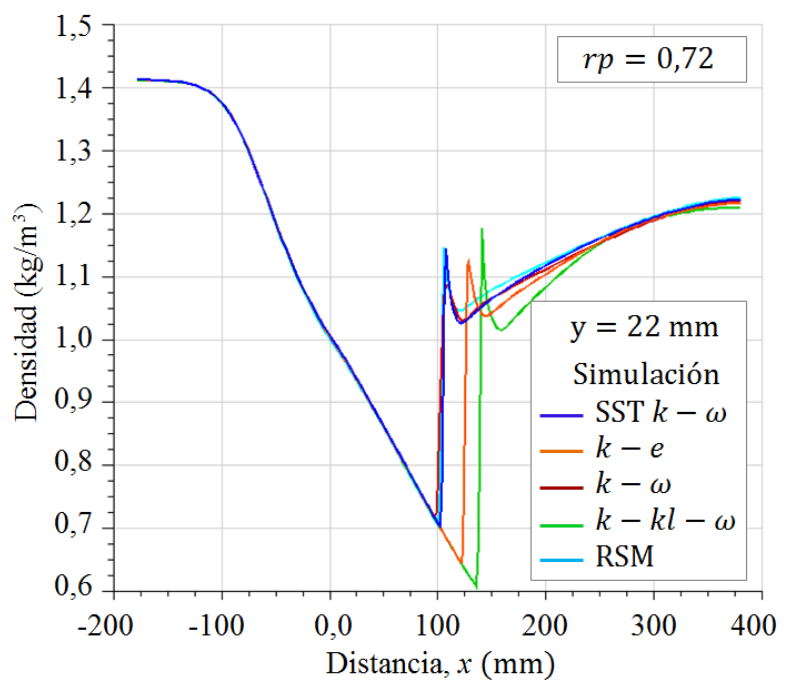

Figura 8. Perfiles de densidad, para el flujo con $\mathrm{rp}=0,72$; obtenido en la altura $\mathrm{y}=22 \mathrm{~mm}$.

La magnitud de la densidad en el instante del inicio y al final del choque, tanto como su posición, para el flujo con $r p=$ 0,82 (Figura 7) se muestra en la Tabla 2, y para el flujo con $r p=0,72$ (Figura 8) se muestra en la Tabla 3. El espesor de la onda de choque se define como $\Delta x=x 2-x 1$, y de la diferencia de densidad $\Delta \mathrm{D}$, para ambos casos del flujo, se presentan en la Tabla 4.

El menor espesor de la onda de choque débil de magnitud $5,280 \mathrm{~mm}$, se obtuvo para SST $k-\omega, k-k l-\omega$ y RSM, siendo de magnitud diferente la densidad; y de mayor magnitud de $12,32 \mathrm{~mm}$ para $k-\omega$. Para el flujo con choque fuerte, los resultados fueron similares para el valor de menor magnitud para el flujo con choque débil, de espesor 5,280 $\mathrm{mm}$, para SST $k-\omega, \quad k-k l-\omega$ y RSM, siendo de magnitud diferente la densidad; y de mayor magnitud 10,556 $\mathrm{mm}$ para $k-\omega$. Aunque los espesores de la onda de choque para algunos modelos de turbulencia son iguales, la magnitud de la densidad durante el choque es diferente, y la posición del inicio y al final del choque también son diferentes, tal como se muestra en la Tabla 2 y 3. 
Las posiciones para las distancias adimensionales, para el flujo con $r p=0,82$ (Figura 7), el inicio del choque se presenta en $x / h_{0}=1,416$ y termina en $x / h_{0}=1,536$; para el flujo con $r p=0,72$ (Figura 8), inicia en $x / h_{0}=2,335$ y termina en $x / h_{0}=2,455$, respectivamente, esto es para la altura $y=22 \mathrm{~mm}$.

Tabla 2. Densidad, para el flujo con $r p=0,82$; para la altura $y=22 \mathrm{~mm}$.

\begin{tabular}{ccccc}
\hline & \multicolumn{2}{c}{ Inicio del choque } & \multicolumn{2}{c}{ Final del choque } \\
& $\boldsymbol{x} \mathbf{1}(\mathbf{m m})$ & $\begin{array}{c}\text { Dens. } \\
\left(\mathbf{k g} / \mathbf{m}^{\mathbf{3}}\right)\end{array}$ & $\boldsymbol{x} \mathbf{2}(\mathbf{m m})$ & $\begin{array}{c}\text { Dens. } \\
\left(\mathbf{k g} / \mathbf{m}^{\mathbf{3}}\right)\end{array}$ \\
\hline SST $k-\omega$ & 62,304 & 0,8243 & 67,584 & 1,1394 \\
$k-e$ & 76,388 & 0,7835 & 83,424 & 1,1316 \\
$k-\omega$ & 69,344 & 0,8034 & 81,664 & 1,1111 \\
$k-k l-\omega$ & 85,184 & 0,7513 & 90,464 & 1,2036 \\
RSM & 72,864 & 0,7864 & 78,144 & 1,1575 \\
\hline
\end{tabular}

Tabla 3. Densidad, para el flujo con $r p=0,72$; para la altura $y=22 \mathrm{~mm}$.

\begin{tabular}{ccccc}
\hline & \multicolumn{2}{c}{ Inicio del choque } & \multicolumn{2}{c}{ Final del choque } \\
& $\boldsymbol{x} \mathbf{1}(\mathbf{m m})$ & $\begin{array}{c}\text { Dens. } \\
\left(\mathbf{k g} / \mathbf{m}^{\mathbf{3}}\right)\end{array}$ & $\boldsymbol{x} \mathbf{2}(\mathbf{m m})$ & $\begin{array}{c}\text { Dens. } \\
\left(\mathbf{k g} / \mathbf{m}^{\mathbf{3}}\right)\end{array}$ \\
\hline SST $k-\omega$ & 102,78 & 0,7039 & 108,06 & 1,1450 \\
$k-e$ & 122,14 & 0,6432 & 129,18 & 1,1242 \\
$k-\omega$ & 97,504 & 0,7182 & 108,06 & 1,0858 \\
$k-k l-\omega$ & 136,22 & 0,6079 & 141,50 & 1,1784 \\
RSM & 101,02 & 0,7038 & 106,30 & 1,1479 \\
\hline
\end{tabular}

Tabla 4. Densidad, para el flujo con $r p=0,82$ y $r p=0,72$; para la altura

\begin{tabular}{ccccc} 
& \multicolumn{4}{c}{$y=22 \mathrm{~mm}}$. \\
\hline & $\Delta \boldsymbol{x}(\mathbf{m m})$ & $\begin{array}{c}\Delta \mathbf{D} \\
\left(\mathbf{k g} / \mathbf{m}^{\mathbf{3}}\right)\end{array}$ & $\begin{array}{c}\boldsymbol{r} \boldsymbol{x}=\mathbf{0}, \mathbf{7 2} \\
(\mathbf{m m})\end{array}$ & $\begin{array}{c}\Delta \mathbf{D} \\
\left(\mathbf{k g} / \mathbf{m}^{3}\right)\end{array}$ \\
\hline SST $k-\omega$ & 5,280 & 0,3151 & 5,280 & 0,4411 \\
$k-e$ & 7,036 & 0,3481 & 7,040 & 0,4810 \\
$k-\omega$ & 12,32 & 0,3077 & 10,556 & 0,3676 \\
$k-k l-\omega$ & 5,280 & 0,4523 & 5,280 & 0,5705 \\
RSM & 5,280 & 0,3711 & 5,280 & 0,4441 \\
\hline
\end{tabular}

La distribución de número de Mach para el flujo con $r p=$ 0,82 (Figura 9) y $r p=0,72$ (Figura 10), fue simulado con el modelo de turbulencia SST $k-\omega$, muestran las regiones donde el flujo es subsónico, transónico y supersónico, así como donde se presenta la onda de choque. Aguas abajo de la onda de choque, en la pared superior, se muestran las regiones donde el flujo es subónico, donde, para $r p=0,72$ se presenta una región con recirculación de flujo.

El comportamiento de la trayectoria del perfil para el número de Mach, para la altura $y=22 \mathrm{~mm}$, se muestra en la Figura 11 para el flujo con $r p=0,82$ y en la Figura 12 para $r p=$ 0,72 . Se observa en ambos perfiles cuando el flujo alcanza un valor máximo y mínimo de número de Mach, donde se produce el choque; en la Tabla 5 se muestran sus magnitudes y la posición al inicio y final del choque. Además, se observa cuando el flujo está en la región subsónica, transónica y supersónica; así como, cuando el flujo alcanza la velocidad sónica, Mach 1.

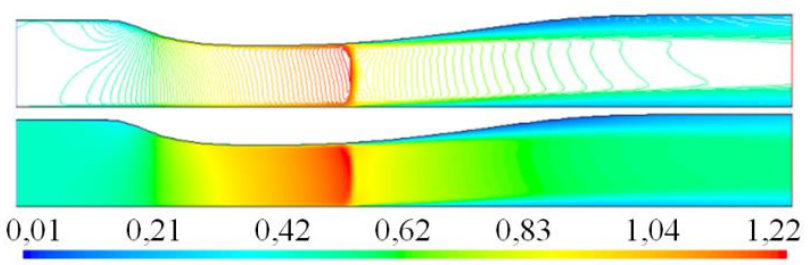

Figura 9. Campo de número de Mach, para el flujo con $r p=0,82$.

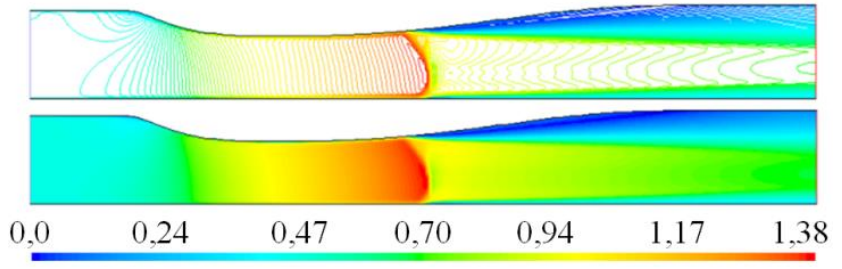

Figura 10. Campo de número de Mach, para el flujo con $\mathrm{rp}=0,72$.

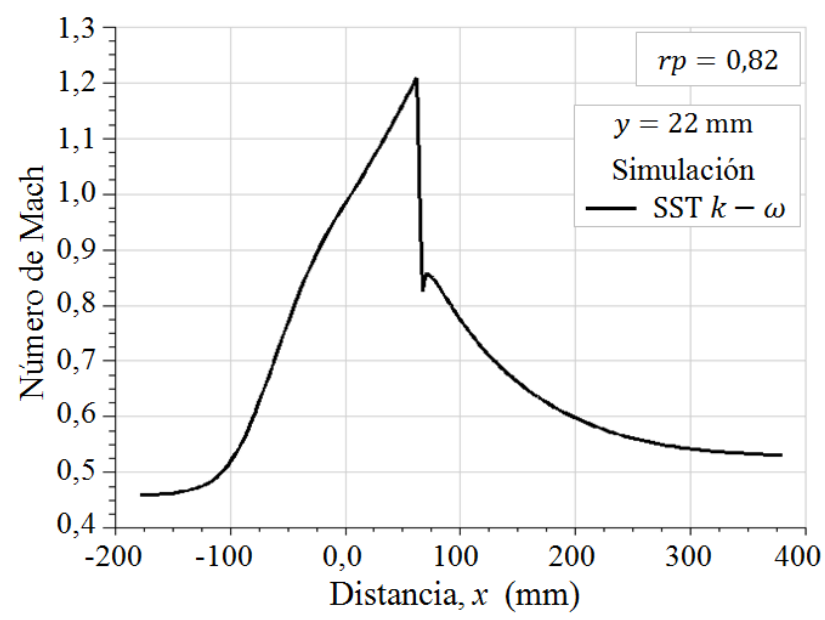

Figura 11. Perfil de número de Mach, para el flujo con $r p=0,82$; obtenido en la altura $\mathrm{y}=22 \mathrm{~mm}$.

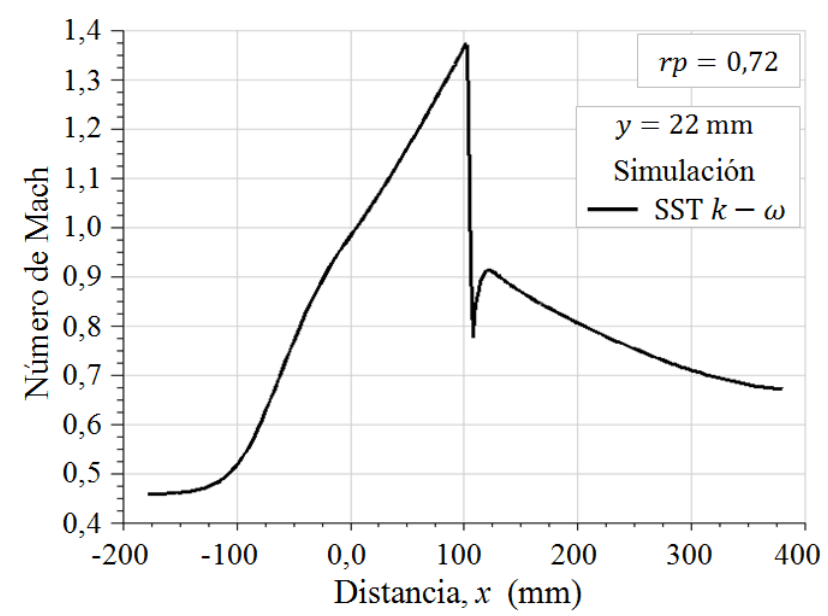

Figura 12. Perfil de número de Mach, para el flujo con $\mathrm{rp}=0,72$; obtenido en la altura $\mathrm{y}=22 \mathrm{~mm}$.

El flujo con choque débil para $r p=0,82$ (Figura 11) alcanza un valor de Mach 1,2105 al inicio del choque, y se desacelera a Mach 0,8264 al final del choque; en la salida del difusor el flujo se desacelera a velocidad subsónica a un valor menor de Mach 0,55. La posición donde se inicia y finaliza el choque es coincidente con la posición para la densidad; por lo tanto, la onda de choque tiene un espesor de magnitud de 5,28 mm, y una variación de número de Mach de 0,3841. Al comparar la magnitud máxima de Mach 1,2105 (Figuras 9 y 11) con respecto al valor de Mach experimental de magnitud 1,235, la cual se muestra en la Figura 13(a), se tiene un error porcentual de $1,9838 \%$.

Así mismo, para el flujo con choque fuerte para $r p=0,72$ (Figura 12), el flujo alcanza un valor de Mach 1,3733 al inicio del choque, y Mach 0,7782 al final del choque, tiene un espesor de 5,28 mm, y una variación de número de Mach de 
0,5951. A la salida del difusor el flujo se desacelera a velocidad subsónica por debajo de Mach 0,7. Al comparar el resultado numérico de Mach 1,3733 (Figuras 10 y 12) con respecto al experimental de Mach 1,353, la cual también se muestra en la Figura 13(b), se tiene un error porcentual de $1,5 \%$.

La imagen de la forma de la onda de choque débil y fuerte obtenido con el modelo de turbulencia SST $k-\omega$, mostradas en las Figuras 5 y 6 para el campo de densidad, así como las Figuras 9 y 10 para el campo de número de Mach, son similares a la forma de la onda de choque experimentales que se muestran en la Figura 13. Además, para el mismo modelo de turbulencia SST $k-\omega$, los perfiles de densidad mostrados en las Figuras 7 y 8 , muestran el comportamiento donde se produce el choque y su posición, así como el comportamiento de la trayectoria del número de Mach mostrados en las Figuras 11 y 12. Para las otras simulaciones del flujo con los otros modelos de turbulencia, mientras mayor o menor sea la diferencia de densidad, y el desplazamiento de su posición, también lo será para el número de Mach, por lo cual, el valor numérico de número de Mach tendrá un valor diferente al valor de Mach experimental. De acuerdo a las comparaciones arriba mencionas, la simulación del flujo con el modelo de turbulencia SST $k-\omega$ se ajustó más al dato experimental del flujo con onda de choque para $r p=0,82$ y $r p=0,72$.

Tabla 5. Número de Mach, para el flujo con choque débil y choque fuerte; para la altura $y=22 \mathrm{~mm}$.

\begin{tabular}{ccccc}
\hline & \multicolumn{2}{c}{ Inicio del choque } & \multicolumn{2}{c}{ Final del choque } \\
& $\boldsymbol{x} \mathbf{1}(\mathbf{m m})$ & Mach & $\boldsymbol{x 2}(\mathbf{m m})$ & Mach \\
\hline$r p=0,82$ & 62,304 & 1,2105 & 67,584 & 0,8264 \\
$r p=0,72$ & 102,78 & 1,3733 & 108,06 & 0,7782 \\
\hline
\end{tabular}
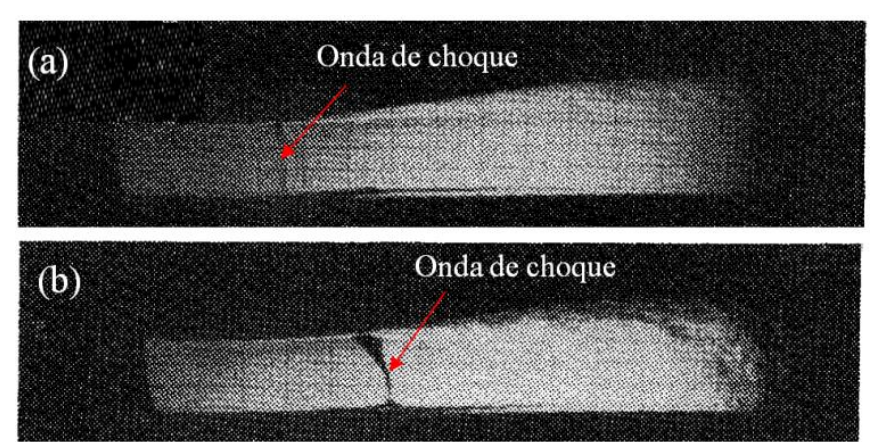

Figura 13. Experimento: imagen captada con la técnica Schlieren. (a) Onda de choque débil: Mach =1,235 para $\mathrm{rp}=0,82$; (b) Onda de choque fuerte: Mach $=1,353$ para rp = 0,72. Fuente: Bogar et al. (1983).

Cabe señalar, los resultados numéricos de los espesores de la onda de choque podrían estar desviados en términos estadísticos para $y=22 \mathrm{~mm}$, sin embargo, aportan información para definir un volumen de control a nivel macroscópico para estudios posteriores; incluso, se puede hacer un análisis aplicando la termodinámica estadística, también para estudios posteriores, ya que en esa región donde se produce el choque el flujo presenta un proceso irreversible, por lo cual, se emplea la ecuación de Rankine-Hugoniot para la solución del problema; y las regiones fuera del choque el proceso es isoentrópico. No obstante, los resultados de los espesores del choque no son concluyentes, y se requiere de datos experimentales para su comparación, de esa manera, tener un criterio más crítico y amplio en el análisis donde se produce el choque.

\subsection{Campo de presión estática}

Las simulaciones numéricas de perfiles de presión estática, empleando los cinco modelos de turbulencia: SST $k-\omega, k-$ $e, k-\omega, k-k l-\omega$ y RSM, para el flujo con relación de presión $r p=0,82$ y $r p=0,72$, se muestran en las Figuras 14 y 15. En ambas figuras, están incorporados los datos experimentales de presiones correspondientes a la pared inferior y superior del difusor transónico Sajben. Cabe resaltar, los datos experimentales de presiones del difusor transónico Sajben fueron tomados del sitio web (Sajben transonic diffuser-NASA), así como, están reportados en el trabajo de Yoder y Georgiadis (1999).

Para la presión del flujo con $r p=0,82$ (Figura 14) y $r p=$ 0,72 (Figura 15), durante la caída e incremento de la presión, las curvas numéricas están superpuestas en ciertos tramos y en otros no, y bordeando los datos experimentales, tanto para la pared superior e inferior. Donde se inicia la separación del flujo, esto es, donde se produce la caída mínima de presión, las que están más alejadas de los datos experimentales son $k-e$ y $k-k l-\omega$. Para el flujo con $r p=0,82$ (Figura 14), en la posición de referencia $x=150 \mathrm{~mm}$, las curvas tienden a unirse; y para $r p=0,72$ (Figura 15) a partir de la posición $x=200 \mathrm{~mm}$.

Para el flujo con $r p=0,82$ (Figura 14) y $r p=0,72$ (Figura 15), las magnitudes de las presiones mínimas y posiciones de los resultados experimentales y numéricos para los cinco modelos de turbulencia empleados, se presentan en un cuadro comparativo: en las Tablas 6 y 7 para el flujo con $r p=0,82$, y en las Tablas 8 y 9 para el flujo con $r p=0,72$, respectivamente.

El dato de la presión experimental para el flujo con $r p=$ 0,82 , en la pared superior (Tabla 6) está ubicado en la posición $x=56,76 \mathrm{~mm}$ con presión de $55081,672 \mathrm{~Pa}$, y en la pared inferior (Tabla 7) en $x=57,2 \mathrm{~mm}$ y presión de $58051,763 \mathrm{~Pa}$; y el que está más se acerca a estos datos es el modelo de turbulencia SST $k-\omega$, en la pared superior (P. sup.) en $x=55,264 \mathrm{~mm}$ con error porcentual de $2,635 \%$ (Tabla 10), y presión de 54737,682 Pa con error porcentual de $5,708 \%$ (Tabla 11); y en la pared inferior (P. inf.) en $x=$ $57,024 \mathrm{~mm}$ con error porcentual de $0,307 \%$ (Tabla 10) y presión de $57486,77 \mathrm{~Pa}$ con error porcentual de $0,973 \%$ (Tabla 11). Por lo tanto, para SST $k-\omega$, el error porcentual en base a la posición es menor de $2,635 \%$, y con respecto a la presión menor de 5,708\%.

Para los otros modelos de turbulencia: $k-e, k-\omega, k-$ $k l-\omega$ y RSM, para el flujo con $r p=0,82$ (Figura 14), el error porcentual con respecto a la posición (Tabla 10) en la pared superior están en el rango de 9,764-37,674\% y en la pared inferior en el rango de 12,0-39,692\%. Con respecto a la presión (Tabla 11) en la pared superior en el rango de 9,345$18,316 \%$ y en la pared inferior en el rango de 4,981-13,339\%, respectivamente. 

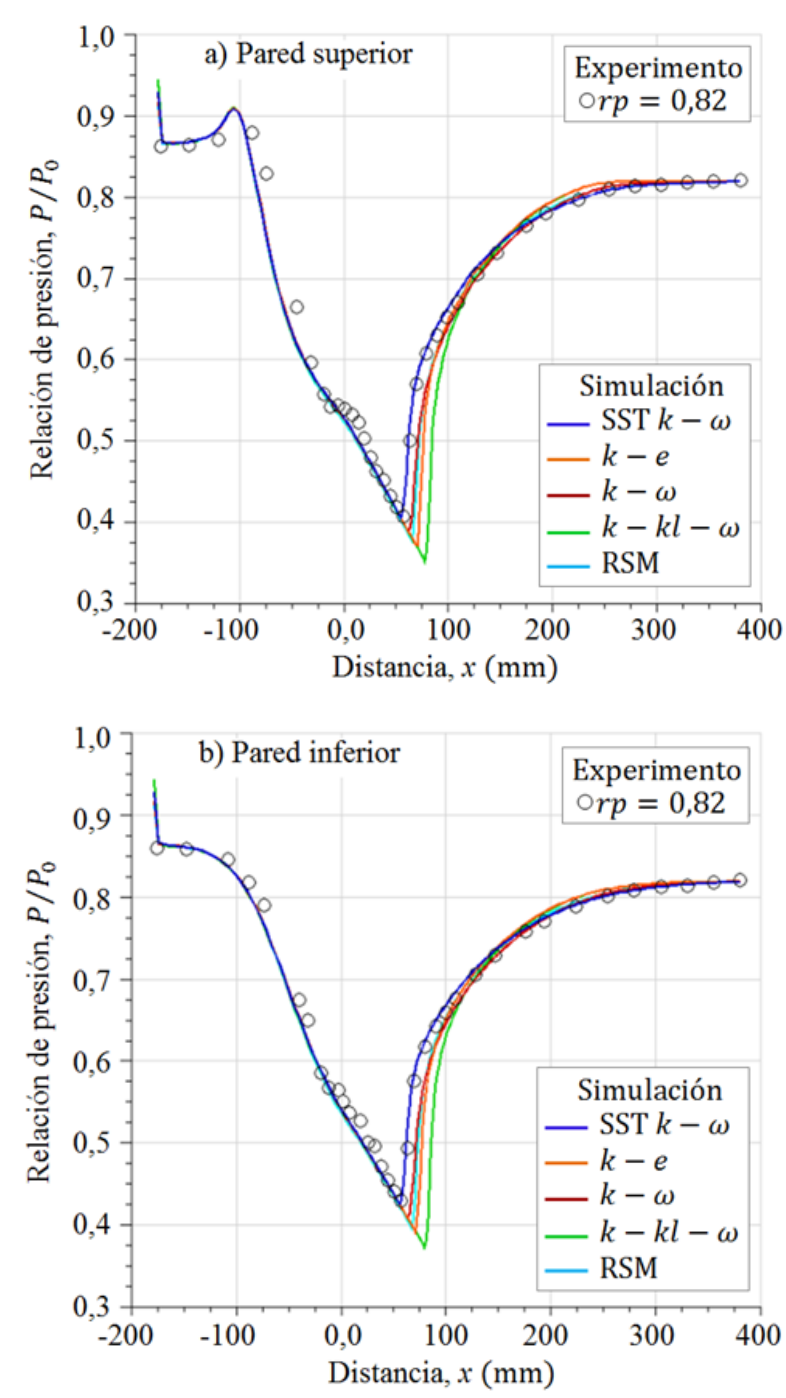

Figura 14. Perfiles de presión estática, para el flujo con $r p=0,82$. a) pared superior. b) Pared inferior.

Al igual que en el caso anterior, se tiene para el flujo con $r p=0,72$ (Figura 15). Donde, en la pared superior (Tabla 8), la presión experimental está ubicado en la posición $x=$ $88,88 \mathrm{~mm}$ con una presión de $46711,418 \mathrm{~Pa}$, y en la pared inferior (Tabla 9) en $x=99,44 \mathrm{~mm}$ y presión de $45226,373 \mathrm{~Pa}$. El resultado del modelo de turbulencia SST $k-\omega$ está más cercano al dato experimental de presión, en la pared superior en $x=85,184 \mathrm{~mm}$ con un error porcentual de 4,158\% (Tabla 10) y presión de 45705,908 Pa con un error porcentual de $1,060 \%$ (Tabla 11); en la pared inferior en $x=95,744 \mathrm{~mm}$ con un error porcentual de $3,716 \%$ (Tabla 10) y presión de $46303,841 \mathrm{~Pa}$ con un error porcentual de 2,382\% (Tabla 11). Así mismo, para SST $k-$ $\omega$, el error porcentual en base a la posición es menor de $4,158 \%$, y con respecto a la presión menor de $2,382 \%$.

Los resultados del modelo de turbulencia RSM, tienen valores casi coincidentes con el modelo SST $k-\omega$, mientras SST $k-\omega$ tiene un error porcentual con respecto a la posición en la pared superior de $4,158 \%$ y en la pared inferior de $3,716 \%$, esto es para el flujo con $r p=0,72$ (Figura 15), el modelo RSM tiene un valor de error porcentual ligeramente mayor, en la pared superior de $4,159 \%$ y en la pared inferior de $3,718 \%$, sin embargo, el error porcentual para las presiones de RSM son menores, en el rango de 0,152-1,081\%, con respecto de SST $k-\omega$ que está en el rango de 1,060$2,382 \%$. Mediante un análisis cualitativo de las curvas numéricas se observa que RSM presenta ligeras fluctuaciones alrededor de los datos experimentales y de SST $k-\omega$.
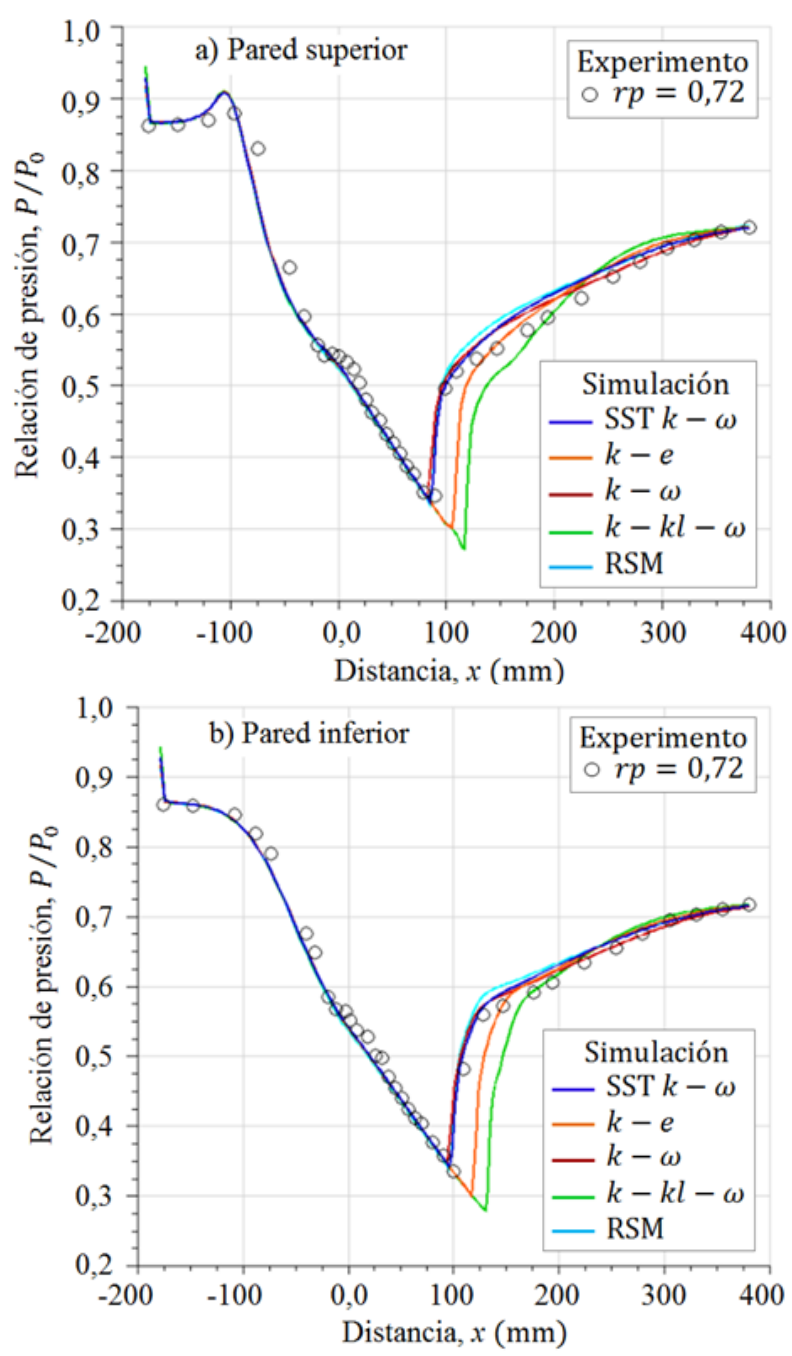

Figura 15. Perfiles de presión estática, para el flujo con $r p=0,72$. a) pared superior. b) Pared inferior.

Los otros modelos de turbulencia: $k-e, k-\omega$ y $k-k l-$ $\omega$, para el flujo con $r p=0,72$ (Figura 15), tienen errores porcentuales con respecto a la posición (Tabla 10), para la pared superior en el rango de $8,118-31,48 \%$ y en la pared inferior en el rango de 5,486-31,677\%; para la presión (Tabla 11 ), en la pared superior en el rango de 3,360-18,331\% y en la pared inferior en el rango de 3,829-16,322\%.

Tabla 6. Datos de posición y de presión en la pared superior.

\begin{tabular}{cccr}
\hline $\boldsymbol{r} \boldsymbol{p}=\mathbf{0 , 8 2}$ & $\boldsymbol{x} \mathbf{( \mathbf { m m } )}$ & $\boldsymbol{P} / \boldsymbol{P}_{\mathbf{0}}$ & $\boldsymbol{P}(\mathbf{P a})$ \\
\hline Experimento: & $\mathbf{5 6 , 7 6}$ & $\mathbf{0 , 4 0 8}$ & $\mathbf{5 5 0 8 1 , 6 7 2}$ \\
\hline SST $k-\omega$ & 55,264 & 0,405452 & 54737,682 \\
$k-e$ & 71,104 & 0,371812 & 50196,144 \\
$k-\omega$ & 62,304 & 0,389813 & 52626,353 \\
$k-k l-\omega$ & 78,144 & 0,351237 & 47418,435 \\
RSM & 65,824 & 0,377098 & 50909,776 \\
\hline
\end{tabular}


Tabla 7. Datos de posición y de presión en la pared inferior.

\begin{tabular}{cccc}
\hline $\boldsymbol{r} \boldsymbol{p}=\mathbf{0 , 8 2}$ & $\boldsymbol{x} \mathbf{( \mathbf { m m } )}$ & $\boldsymbol{P} / \boldsymbol{P}_{\mathbf{0}}$ & $\boldsymbol{P}(\mathbf{P a})$ \\
\hline Experimento: & $\mathbf{5 7 , 2 0}$ & $\mathbf{0 , 4 3 0}$ & $\mathbf{5 8 0 5 1 , 7 6 3}$ \\
\hline SST $k-\omega$ & 57,024 & 0,425815 & 57486,770 \\
$k-e$ & 71,104 & 0,391137 & 52805,098 \\
$k-\omega$ & 64,064 & 0,408581 & 55160,110 \\
$k-k l-\omega$ & 79,904 & 0,372639 & 50307,792 \\
RSM & 67,584 & 0,396565 & 53537,900 \\
\hline
\end{tabular}

Tabla 8. Datos de posición y de presión en la pared superior.

\begin{tabular}{cccr}
\hline $\boldsymbol{r} \boldsymbol{p}=\mathbf{0 , 7 2}$ & $\boldsymbol{x}(\mathbf{m m})$ & $\boldsymbol{P} / \boldsymbol{P}_{\mathbf{0}}$ & $\boldsymbol{P}(\mathbf{P a})$ \\
\hline Experimento: & $\mathbf{8 8 , 8 8}$ & $\mathbf{0 , 3 4 6}$ & $\mathbf{4 6 7 1 1 , 4 1 8}$ \\
\hline SST $k-\omega$ & 85,184 & 0,338552 & 45705,908 \\
$k-e$ & 106,30 & 0,302356 & 40819,299 \\
$k-\omega$ & 81,664 & 0,346256 & 46745,979 \\
$k-k l-\omega$ & 116,86 & 0,273588 & 36935,501 \\
RSM & 85,183 & 0,334489 & 45157,386 \\
\hline
\end{tabular}

Tabla 9. Datos de posición y de presión en la pared inferior.

\begin{tabular}{cccr}
\hline $\boldsymbol{r} \boldsymbol{p}=\mathbf{0}, \mathbf{7 2}$ & $\boldsymbol{x}(\mathbf{m m})$ & $\boldsymbol{P} / \boldsymbol{P}_{\mathbf{0}}$ & $\boldsymbol{P}(\mathbf{P a})$ \\
\hline Experimento: & $\mathbf{9 9 , 4 4}$ & $\mathbf{0 , 3 3 5}$ & $\mathbf{4 5 2 2 6 , 3 7 3}$ \\
\hline SST $k-\omega$ & 95,744 & 0,342981 & 46303,841 \\
$k-e$ & 116,86 & 0,300379 & 40552,396 \\
$k-\omega$ & 93,984 & 0,347829 & 46958,341 \\
$k-k l-\omega$ & 130,94 & 0,280320 & 37844,349 \\
RSM & 95,742 & 0,338622 & 45715,358 \\
\hline
\end{tabular}

Tabla 10. Error porcentual con respecto a la posición experimental.

\begin{tabular}{ccccc}
\hline & \multicolumn{3}{c}{ Posición: error porcentual, \% } \\
& P. sup. & P. $\mathbf{~} 2$ & \multicolumn{2}{c}{$\boldsymbol{r p}=\mathbf{0 , 7 2}$} \\
& 2,635 & 0,307 & P. sup. & P. inf. \\
\hline SST $k-\omega$ & 25,271 & 24,307 & 19,599 & 3,716 \\
$k-e$ & 9,764 & 12,000 & 8,118 & 17,518 \\
$k-\omega$ & 37,674 & 39,692 & 31,48 & 31,677 \\
$k-k l-\omega$ & 15,968 & 18,153 & 4,159 & 3,718 \\
RSM & \multicolumn{4}{c}{}
\end{tabular}

Tabla 11. Error porcentual con respecto a la caída de presión.

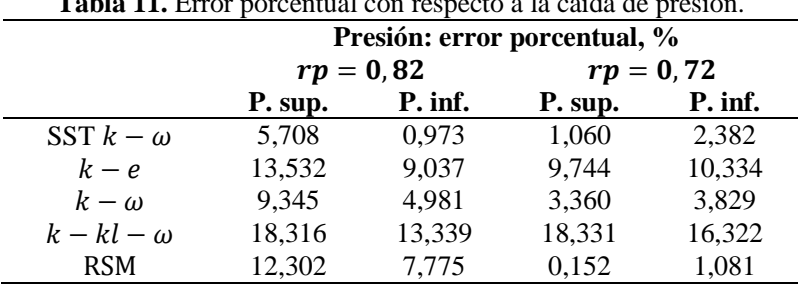

Del análisis realizado, el modelo de turbulencia de SST $k-$ $\omega$, es el que más se ajustó a los datos experimentales de presión.

En las Figuras 16 y 17 se muestra la distribución de presión estática en diferentes regiones del campo de flujo, así como, en las regiones donde se presenta las ondas de choque. Para la altura $y=22 \mathrm{~mm}$, al inicio y final de la onda de choque, para el flujo con $r p=0,82$, la relación de presión $P / P_{0}$ está en el rango estimado de $0,4-0,64$, y para el flujo con $r p=$ 0,72 en el rango de $0,33-0,65$, respectivamente.

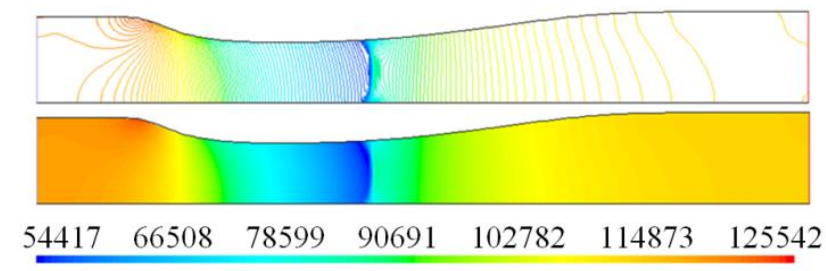

Figura 16. Campo de presión estática $(\mathrm{Pa})$, para el flujo con $r p=0,82$.

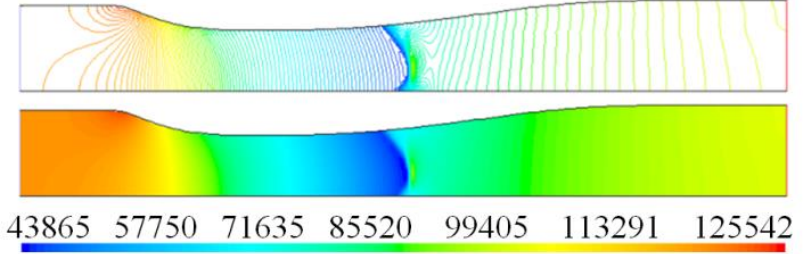

Figura 17. Campo de presión estática $(\mathrm{Pa})$, para el flujo con $\mathrm{rp}=0,72$.

Cabe resaltar, se refinó la malla a lo largo de la pared para reducir a la mínima expresión los errores numéricos para la presión, en las paredes del difusor, por lo cual, la densidad de la malla se obtuvo para $y^{+}<1$, para la simulación del flujo en un dominio computacional 2D. Sin embargo, se debe simular el campo de flujo de presión para un dominio 3D, y determinar el comportamiento de la presión en las paredes y comparar los resultados experimentales, así mismo, comparar con los resultados del presente trabajo.

\subsection{Campo de velocidad}

Los perfiles de velocidad del flujo para diferentes tramos a lo largo del difusor transónico, para $x / h_{0}=2,882, x / h_{0}=$ $4,611, x / h_{0}=6,34$ y $x / h_{0}=7,493$, obtenidos para los cinco modelos de turbulencia: SST $k-\omega, k-e, k-\omega, k-$ $k l-\omega$ y RSM, para el flujo con $r p=0,82$ y $r p=0,72$, son comparados con datos experimentales de velocidad, las cuales se muestran en las Figuras 18 y 19. Donde, los datos experimentales de velocidad están reportados en el sitio web (Sajben transonic diffuser-NASA), así como, en el trabajo Yoder y Georgiadis (1999).

La posición de la velocidad del flujo para $r p=0,82$ (Figura 18) y $r p=0,72$ (Figura 19), inicia a partir de $x / h_{0}=2,882$, lo cual corresponde una posición aguas abajo después del choque. Ya que, la posición final del choque se presenta en $x / h_{0}=1,536$ para $r p=0,82$, y en $x / h_{0}=2,455$ para $r p=0,72$, respectivamente. Para el flujo con $r p=0,82$ (Figura 18), se muestra que la curva para el modelo de turbulencia SST $k-\omega$ tiene mayor acercamiento a los datos experimentales de velocidad, realizando una comparación cualitativa. Además, se observa el comportamiento de la trayectoria del perfil, en el frente del desarrollo del flujo hacia aguas abajo, iniciando por $x / h_{0}=2,882, x / h_{0}=$ $4,611, x / h_{0}=6,34$ y $x / h_{0}=7,493$, así como, para la región del flujo que está adyacente a la pared inferior; en la pared superior, el perfil numérico se aleja de los datos experimentales, iniciando por $x / h_{0}=4,611, x / h_{0}=6,34 \mathrm{y}$ $x / h_{0}=7,493$. Sin embargo, los perfiles que más predominan en las cercanías de los datos experimentales para la región del flujo cercano a las paredes son para $k-e, k-$ $\omega, k-k l-\omega$ y RSM, y el que tiene mayor contacto es $k-$ $e$. 

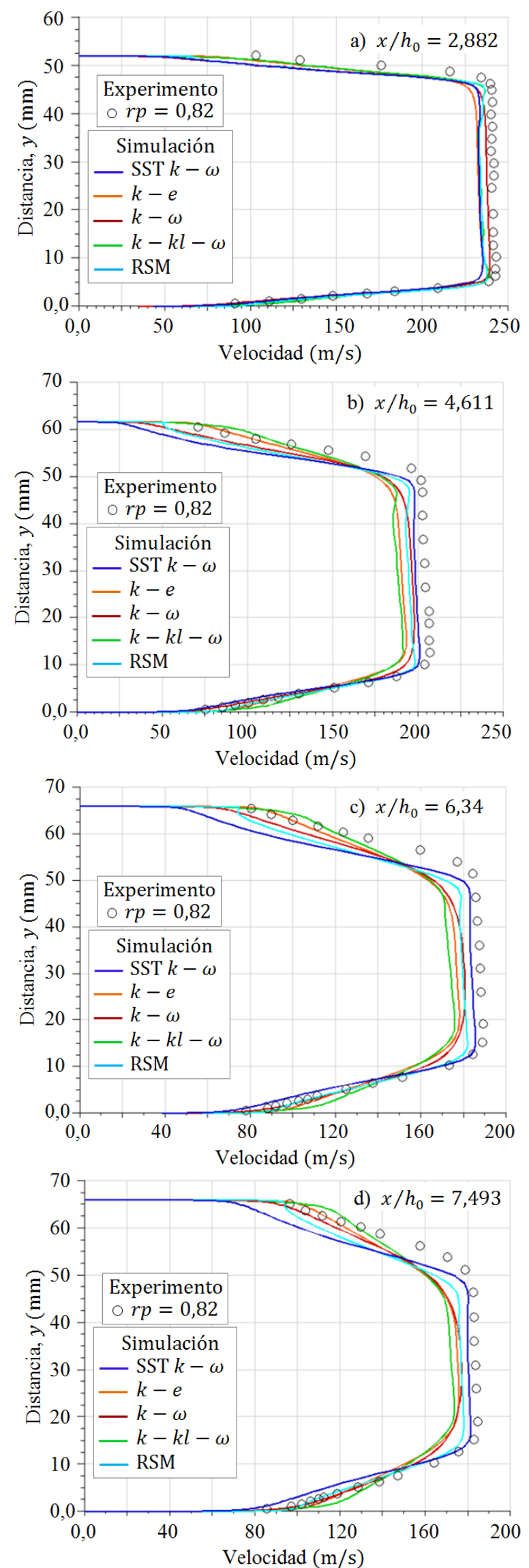

Figura 18. Perfiles de velocidad, para el flujo con $r p=0,82$.
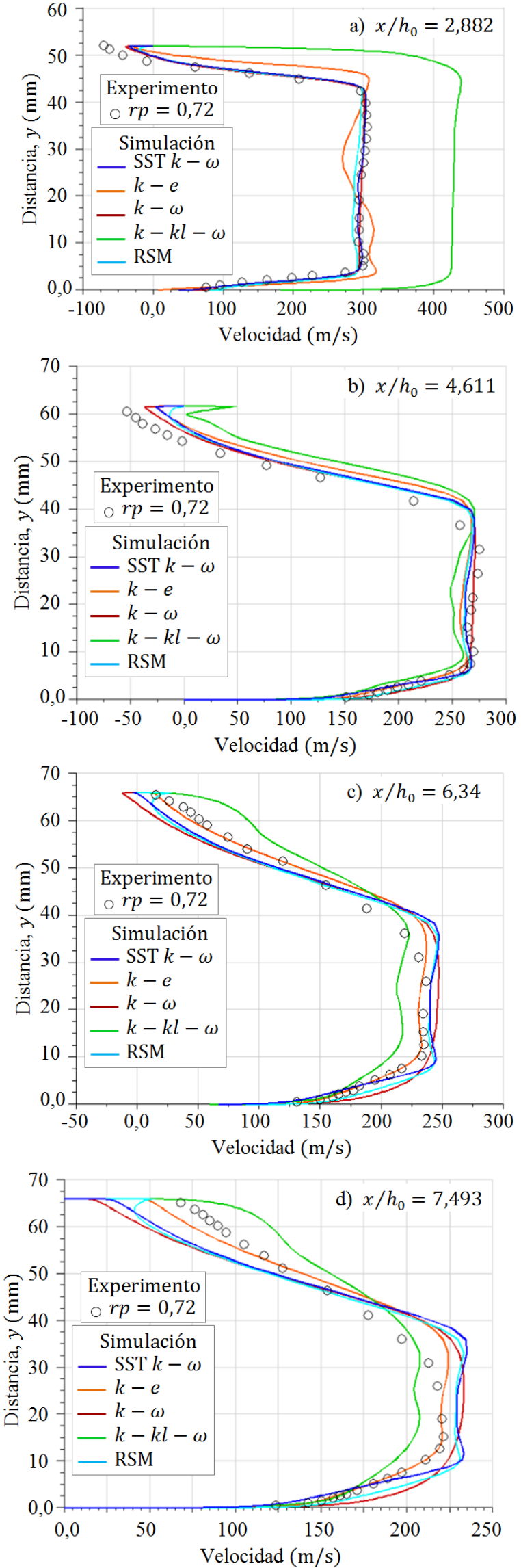

Figura 19. Perfiles de velocidad, para el flujo con $r p=0,72$. 
Así mismo, para el flujo con $r p=0,72$ (Figura 19), el que más se ajusta a los datos experimentales de velocidad en $x / h_{0}=2,882$ y $x / h_{0}=4,611$, es SST $k-\omega$. Las curvas $k-\omega$ y RSM tienen ciertos tramos superpuestas y otros tramos cercanas a SST $k-\omega$. Las curvas $k-e$ y $k-k l-$ $\omega$ tiene un comportamiento oscilatorio en el frente del flujo, y el que está más alejado es $k-k l-\omega$ para $x / h_{0}=2,882$. Para las posiciones $x / h_{0}=6,34$ y $x / h_{0}=7,493$, el que tiene mayor acercamiento a los datos experimentales es $k-$ $e$; las curvas SST $k-\omega$ y RSM tiene un comportamiento similar entre sí, y los que están más alejados son $k-\omega$ y $k-$ $k l-\omega$.

Tanto para el flujo con onda de choque débil para $r p=0,82$ (figura 18) y choque fuerte para $r p=0,72$ (Figura 19), el modelo de turbulencia SST $k-\omega$ se ajustó más a los datos experimentales de velocidad. En Las Figuras 20 y 21, se muestran las distribuciones de velocidad en todo el campo de flujo simulado con SST $k-\omega$, y se aprecia donde se presenta las ondas de choque. Para la altura $y=22 \mathrm{~mm}$, el inicio y final de la onda de choque, para el flujo con $r p=0,82$, la velocidad está en el rango estimado de $270-370(\mathrm{~m} / \mathrm{s})$, y para el flujo con $r p=0,72$ en el rango de $251-405(\mathrm{~m} /$ s).

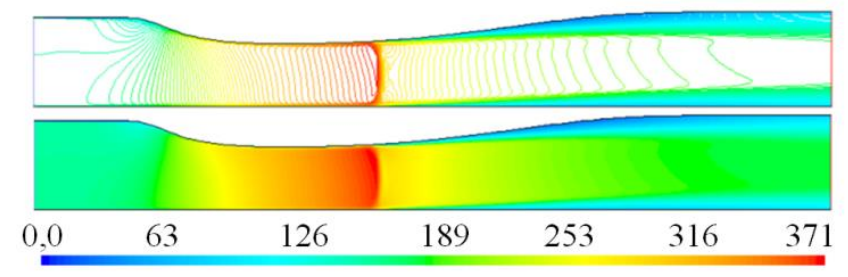

Figura 20. Campo de velocidad $(\mathrm{m} / \mathrm{s})$, para el flujo con $r p=0,82$.

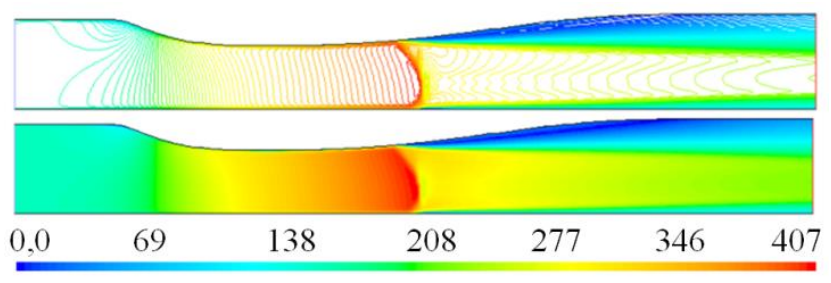

Figura 21. Campo de velocidad (m/s), para el flujo con $\mathrm{rp}=0,72$.

Cabe destacar, en la pared superior, para la posición $x / h_{0}=$ 2,882 y $x / h_{0}=4,611$, una región del flujo adyacente a la pared presenta una recirculación del flujo, tal como lo demuestran ciertos tramos cortos de las curvas de los modelos de turbulencia con magnitud de velocidad negativa, además, los datos experimentales de velocidad corroboran que existe una velocidad negativa del flujo, por lo cual el flujo en esa región va en sentido contrario, ocasionado por la presencia de un remolino. Para la posición $x / h_{0}=6,34$, disminuye la intensidad de la velocidad negativa, y para la posición $x / h_{0}=7,493$ ya no se presenta una velocidad negativa por lo cual, a partir de esta última posición, la región del flujo adyacente a la pared ya no presenta remolinos que ocasionen recirculación del flujo. La región central del flujo para las posiciones $x / h_{0}=6,34$ y $x / h_{0}=7,493$ debe ser una región de interés para analizar con más detalle sobre las fluctuaciones del flujo, y se debe considerar como una región crítica. La posición $x / h_{0}=7,493$ corresponde para $x=$
329,692 mm y está cercano a la salida del difusor transónico que termina en la posición $x / h_{0}=8,65$ en $x=380,6 \mathrm{~mm}$.

En trabajo a futuro, se debe considerar la simulación de la velocidad del flujo en un dominio $3 \mathrm{D}$, empleando los cinco modelos de turbulencia, y corroborar con los resultados numéricos para el dominio $2 \mathrm{D}$ del presente trabajo, para las posiciones de velocidad en $x / h_{0}=6,34$ y $x / h_{0}=7,493$.

\subsection{Campo de temperatura estática}

La simulación del campo de temperatura estática para el flujo con $r p=0,82$ (Figura 22) y $r p=0,72$ (Figura 23), se obtuvo con el modelo de turbulencia SST $k-\omega$. Se muestra cómo se distribuye el gradiente de temperatura en el campo de flujo, antes y después que se presenta la onda de choque. Inducido por a la geometría de las paredes del difusor transónico, el flujo se ve influenciado a pasar a mayor velocidad por la parte más estrecha, ocasionando la disminución de la presión, y la expansión de la densidad, por lo cual, ocasiona la disminución de la temperatura; luego de la onda de choque, la presión se incrementa así como la densidad, así mismo la temperatura. Sin embargo, donde se inicia la separación del flujo después de la onda de choque, la temperatura del flujo tiene mayor magnitud con respecto al resto del flujo, así como, aguas abajo hasta la salida del difusor. Señalando que, la temperatura estática es la que existe sólo en virtud de la energía interna.

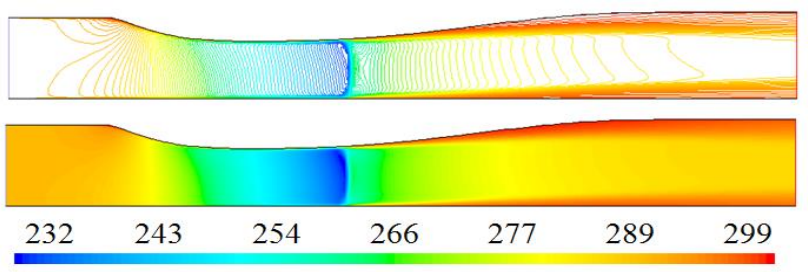

Figura 22. Campo de temperatura estática $(K)$, para el flujo con $r p=0,82$.

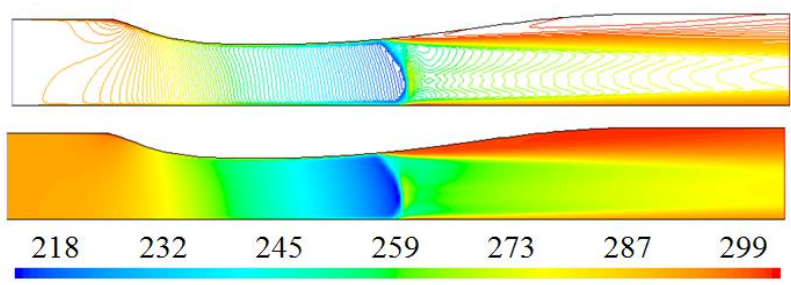

Figura 23. Campo de temperatura estática $(\mathrm{K})$, para el flujo con $\mathrm{rp}=0,72$.

Los perfiles de temperatura estática en la dirección $x$ se muestran en las Figuras 24 y 25, para el flujo con $r p=0,82$ y $r p=0,72$, y se muestra el comportamiento de la trayectoria de cada perfil. En la pared superior e inferior, considerados paredes adiabáticas, la temperatura disminuye levemente, y en el inicio de la separación del flujo se incrementa, el cual se encuentra en el rango estimado de $290-300 \mathrm{~K}$. Para las regiones del flujo que están distanciado de la pared, la temperatura disminuye drásticamente por la caída de presión y la expansión del flujo en esa región, hasta el inicio del choque, y se presenta la menor caída para $y=22 \mathrm{~mm}$. Al final del choque la temperatura se incrementa producto de la desaceleración brusca del flujo e incremento abrupto de la compresión, siendo su magnitud menor a la temperatura de las regiones del flujo que están en contacto con las paredes. 
Para la altura $y=22 \mathrm{~mm}$, para el flujo con $r p=0,82$, entre el inicio y final del choque, la temperatura estática está en el rango estimado de $232-265 \mathrm{~K}$; para el flujo con $r p=0,72$, en el rango estimado de $217-267 \mathrm{~K}$. Por lo cual, para el flujo con choque fuerte se produce menor caída de temperatura.

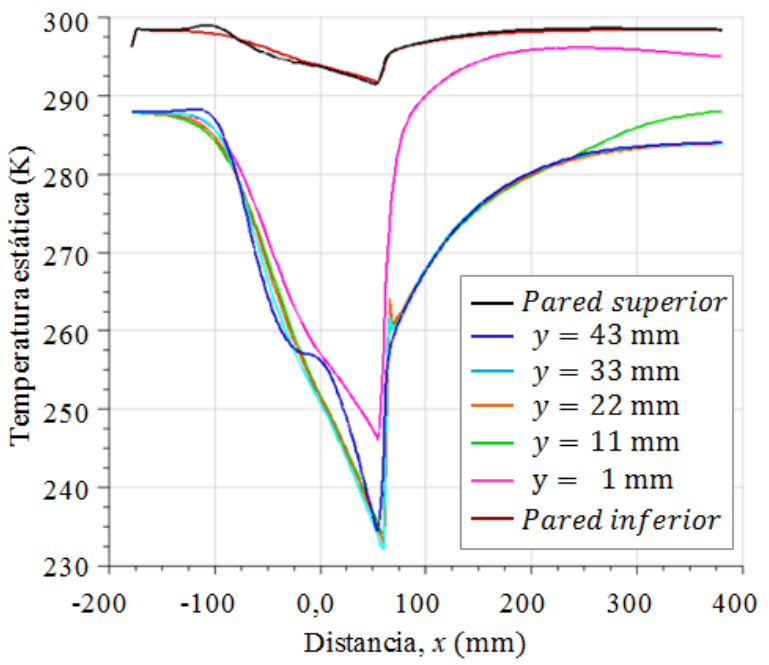

Figura 24. Perfiles de temperatura estática, para el flujo con $\mathrm{rp}=0,72$; obtenido para diferentes alturas en $\mathrm{y}$.

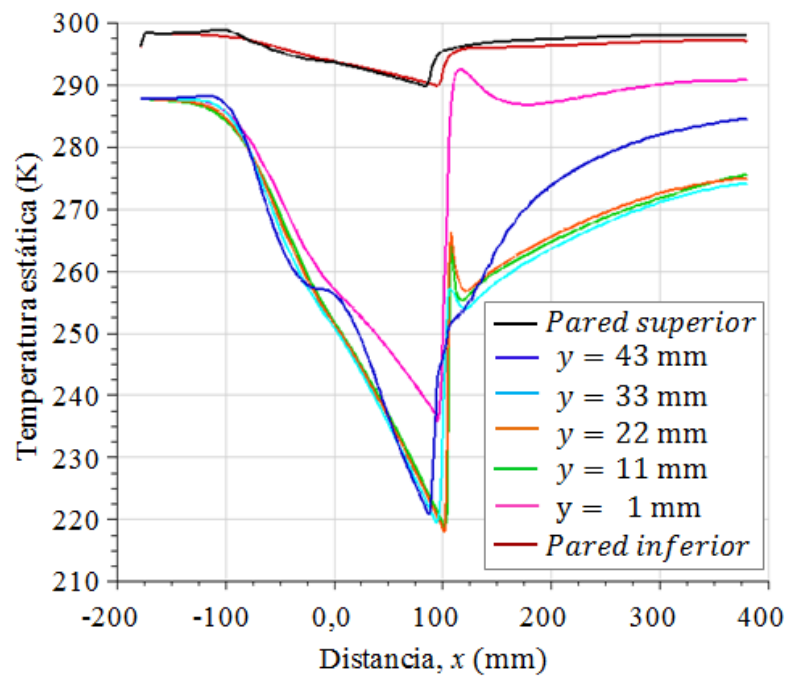

Figura 25. Perfiles de temperatura estática, para el flujo con $\mathrm{rp}=0,72$; obtenido para diferentes alturas en $\mathrm{y}$.

\section{CONCLUSIONES}

En base a los resultados obtenidos de la simulación del flujo para los cinco modelos de turbulencia: SST $k-\omega, k-e$ estándar, $k-\omega$ estándar, $k-k l-\omega$ de transición y RSM, los cuales han sido comparados con datos experimentales, se concluye que:

Los resultados numéricos obtenidos tienen que ver con los fundamentos matemáticos de cada modelo de turbulencia y el método de evaluación que aplican en la región dentro y fuera de la capa límite. Ya que, dentro de la capa límite, para la región del flujo adyacente a la pared están presente el esfuerzo cortante; y existen dos parámetros de interés, el espesor y el coeficiente de fricción, tanto para flujo laminar o turbulento. Los modelos de turbulencia tienen divergencias en los resultados numéricos cuando se produce la separación del flujo de la pared, pues, seguidamente del punto de separación, está presente una región del flujo con sentido inverso, presentando velocidades negativas. Cuando son comparados con datos experimentales de presión y de velocidad, ciertos tramos de las curva numéricas están próximas y otras alejadas de los datos experimentales. Por lo cual, se considera que se debe evaluar los cinco modelos de turbulencia para un dominio $3 \mathrm{D}$, de esa manera, tener un criterio de evaluación más amplio con respecto al desarrollo del flujo después que se presenta el choque. Sin embargo, de los cinco modelos de turbulencia empleados, el que más se ajusta a los datos experimentales de densidad, número de Mach, presión y velocidad, es el modelo de turbulencia SST $k-\omega$ de Menter.

Además, en base a los resultados obtenidos con el modelo de turbulencia SST $k-\omega$, para la altura $y=22 \mathrm{~mm}$, se concluye que:

El espesor de la onda de choque para el flujo con $r p=0,82$, presenta un espesor de $5,28 \mathrm{~mm}$ y la variación de densidad de $0,3151\left(\mathrm{~kg} / \mathrm{m}^{3}\right)$; y para el flujo con $r p=0,72$, el espesor de 5,28 mm y la variación de densidad de $0,4411\left(\mathrm{~kg} / \mathrm{m}^{3}\right)$.

Para el caso de número de Mach, que fue comparado el resultado numérico con el experimental, al inicio del choque para el flujo con $r p=0,82$, se obtuvo un error porcentual de $1,9838 \%$; y para el flujo con $r p=0,72$ de $1,5 \%$.

Para la presión, al inicio y final de la onda de choque, para el flujo con $r p=0,82$, la relación de presión $P / P_{0}$ está en el rango estimado de $0,4-0,64$, y para el flujo con $r p=0,72$ en el rango de 0,33-0,65. En las paredes donde se inicia la separación de flujo, la presión estática al ser comparado con datos experimentales para el flujo con $r p=0,82$, en la pared superior el error porcentual fue de $5,708 \%$ y en la pared inferior de $0,973 \%$. Así mismo, para el flujo con $r p=0,72$, en la pared superior el error porcentual fue de $1,060 \%$ y en la pared inferior de $2,382 \%$.

La velocidad el flujo, el inicio y final de la onda de choque, para el flujo con $r p=0,82$, se obtuvo que la velocidad está en el rango estimado de $270-370(\mathrm{~m} / \mathrm{s})$, y para el flujo con $r p=0,72$ en el rango de $251-405(\mathrm{~m} / \mathrm{s})$.

Para la temperatura estática, para el flujo con $r p=0,82$, entre el inicio y final del choque, se obtuvo la temperatura en el rango estimado de $232-265 \mathrm{~K}$; para el flujo con $r p=$ 0,72 , en el rango estimado de $217-267 \mathrm{~K}$. Por lo cual, para el flujo con choque fuerte se produce menor caída de temperatura.

\section{AGRADECIMIENTOS}

Mi agradecimiento a Jehová, mi Dios todopoderoso, mi fuente de sabiduría e inspiración. Al Centro de Estudios Energéticos de la Universidad Nacional Experimental Politécnica "Antonio José de Sucre" Vice-Rectorado Puerto Ordaz, Bolívar, Venezuela. Al Grupo de Modelamiento 
Matemático y Simulación Numérica (GMMNS, Group of Mathematical Modeling and Numerical Simulation) de la Universidad Nacional de Ingeniería (UNI), Lima, Perú.

\section{REFERENCIAS}

Anderson, J. (2007). Fundamentals of aerodynamics. Fifth Edition, Mc Graw-Hill, USA.

Anderson, B., Anderson, R., Hakansson, L., Mortensen, L., Sudiyo, R., Wachem, B. (2012). Computational fluid dynamics for engineers. Cambridge university press.

Biswas, D., Jimbo, T. (2015). Studies on characteristics frequency and length scale of shock induced motion in transonic diffuser using a high order les approach. ASME GTINDIA Conference, december 2-3, Hyderabad, India.

Blazek, J. (2015). Computational fluid dynamics: principles and applications. Third Edition, Elsevier.

Bogar, T.J., Sajben, M., Kroutill, J.C. (1983). Characteristic frecuencies of transonic diffuser flow oscillations. AIAA Journal, 21(9), 1232-1240.

Bogar, T.J. (1986). Structure of self-excited oscillations in transonic diffuser flows. AIAA Journal, 24(1), 54-61.

Bush, R.H., Power, G.D., Towne, C.E. (1998). WIND: the production flow solver of the NPARC Alliance. AIAA 98-0935, 36th Aerospace Sciences Meeting exhibit, January 12-15, Reno, NV.

Georgiadis, N.J., Drummod, J.E., Leonard, B.P. (1994). Evaluation of turbulence models in the PARC code for transonic diffuser flows. AIAA 94-0582, NASA TM 106391.

Gibson, M.M., Launder, B. (1978). Ground effects on pressure fluctuations in the atmospheric boundary layer. Journal Fluid Mech, 86, 491-511.

Hsieh, T., Wardlaw, A.B., Collins, P. (1987). Numerical investigation of unsteady inlet flow fields. AIAA Journal, 25(1), 75-81.

Kármán, Th. (1937). The fundamentals of the statistical theory of turbulence. Journal of the Aeronautical Sciences, 4(4), 131-138.

Krehl, P., Engemann, S. (1995). August Toepler the first who visualized shock waves. Journal Shock Waves, 5, 1-18.

Kolmogorov, N.P. (1941). The local structure of turbulence in incompressible viscous fluid for very large Reynolds numbers. Academy of Sciences of the USSR, 30, 299-303.

Launder, B.E., Spalding, D.B. (1972). Lectures in mathematical models of turbulence. Academic Press, London, England, 169-189.

Launder, B., Reece, G.J., Rodi, W. (1975). Progress in the development of a Reynolds-stress turbulence closure. J. Journal of Fluid Mechanics, 68(3), 537-566.

Launder, B. (1989). Second-moment closure and its use in modeling turbulent industrial flows. Internationa Journal for Numerical Methods in Fluids. 9, 963-985.

Liu, Y., Wu, J., Lu, L. (2016). Performance of turbulence models for transonic flows in a diffuser. World Scientific, 30(25) 1650326, 1-14 DOI: $10.1142 / \mathrm{S} 0217984916503267$.

Liou, M.S., Coakley, T.J, Bergmann, M.Y. (1981). Numerical simulation of transonic flows in diffusers. AIAA 14th Fluid and plasma dynamics conference, June 23-25, California.

Menter, F. (1994). Two equation eddy-viscosity turbulence models for engineering applications. AIAA Journal, 32, 1598-1605.

Sajben transonic diffuser-NASA. Obtenido de https://www.grc.nasa.gov/www/wind/valid/transdif/transdif.html. (Diciembre, 2019).

Sajben, M., Bogar, T.J., Kroutil J.C. (1981). Forced oscillation experiments in supercritical diffuser flows with application to ramjet instabilities. AIAA/SAE/ASME, 17th Join Propulsion Conference, July 27-29, Colorado.

Sajben, M., Bogar, T.J., Kroutil, J.C. (1982). Unsteady transonic flows in a two-dimensional diffuser. AFOSR-TR-82-0622, Report MDC Q0767, 1-17.

Salmon, J.T., Bogar, T.J., Sajben, M. (1983). Laser doppler velocimeter measurements in unsteady, separated, transonic diffuser flows. AIAA Journal, 21(12), 1690-1697.

Schlichting, H. (1979). Boundary-layer theory. Seventh Edition, Mc GrawHill, USA.

Tolentino, S.L. y Caraballo, S. (2017). Simulación numérica del flujo de aire con onda de choque en un difusor transónico. Revista Universidad, Ciencia y Tecnología (UCT), 21(82), 4-15.

Vlahostergios, Z., Yakinthos, K. (2015). Modelling the flow in a transonic diffuser with one Reynlods-stress and two eddy-viscosity models. Flow Turbulence Combust, Springer, DOI: 10.1007/s10494-015-95962.
Walters, D.K., Cokljat D. (2008). A three-equation eddy-viscosity model for Reynolds-averaged Navier-Stokes simulations of transitional flows. Journal of Fluids Engineering, 130, 121401 1-14.

White, F. (1991). Viscous fluid flow. Second Edition, Mc Graw-Hill, USA.

White, F. (2008). Mecánica de los Fluidos. Sexta Edición, Mc Graw-Hill, Interamericana de España.

Wilcox, D. (1988). Reassessment of the scale-determining equation for advanced turbulence models. AIAA Journal, 26(11), 1299-1310.

Wilcox, D. (2006). Turbulence modeling for CFD. Third Edition, DCW Industries, USA.

Wüthrich, B. (2007). Simulation and validation of compressible flow in nozzle geometries and validation of openFOAM for this application. Master Thesis SS 07, Institute of Fluid Dynamics ETH Zurich.

Xiao, Q., Tasai, H. (2003). Computation of transonic diffuser flows by a lagged $\mathrm{k}-\omega$ turbulence model. Journal of Propulsion and Power, 19(3), 473-483.

Yoder, D.A., Georgiadis, N.J. (1999). Implementation and validation of the Chien k-e turbulence model in the wind Navier-Stokes code. AIAA-990745, NASA TM 1999-209080.

\section{BIOGRAFÍA}

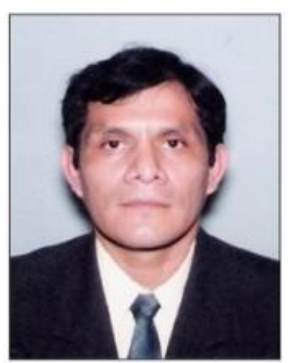

San Luis B. Tolentino Masgo. Ingeniero Mecánico y Magister Scientiarum en Ingeniería Mecánica, egresado de la Universidad Nacional Experimental Politécnica "Antonio José de Sucre" Vice-Rectorado Puerto Ordaz, Bolívar, Venezuela. Sus trabajos están orientados en la línea de investigación: Modelación y Simulación Computacional. A la fecha, cursa estudios de Doctorado en Ciencias de la Ingeniería en la misma universidad. Es investigador colaborador del Centro de Estudios Energéticos (CEE) de la Universidad Nacional Experimental Politécnica "Antonio José de Sucre" ViceRectorado Puerto Ordaz, Bolívar, Venezuela; y del Group of Mathematical Modeling and Numerical Simulation (GMMNS) de la Universidad Nacional de Ingeniería (UNI), Lima, Perú. 